\title{
Black Strings, Black Rings and State-space Manifold
}

\author{
Stefano Bellucci ${ }^{a *}$ and Bhupendra Nath Tiwari ${ }^{a} \dagger$ \\ ${ }^{a}$ INFN-Laboratori Nazionali di Frascati \\ Via E. Fermi 40, 00044 Frascati, Italy.
}

\begin{abstract}
State-space geometry is considered, for diverse three and four parameter non-spherical horizon rotating black brane configurations, in string theory and $M$-theory. We have explicitly examined the case of unit Kaluza-Klein momentum $D_{1} D_{5} P$ black strings, circular strings, small black rings and black supertubes. An investigation of the state-space pair correlation functions shows that there exist two classes of brane statistical configurations, viz., the first category divulges a degenerate intrinsic equilibrium basis, while the second yields a non-degenerate, curved, intrinsic Riemannian geometry. Specifically, the solutions with finitely many branes expose that the two charged rotating $D_{1} D_{5}$ black strings and three charged rotating small black rings consort real degenerate state-space manifolds. Interestingly, arbitrary valued $M_{5}$-dipole charged rotating circular strings and Maldacena Strominger Witten black rings exhibit non-degenerate, positively curved, comprehensively regular state-space configurations. Furthermore, the state-space geometry of single bubbled rings admits a well-defined, positive definite, everywhere regular and curved intrinsic Riemannian manifold; except for the two finite values of conserved electric charge. We also discuss the implication and potential significance of this work for the physics of black holes in string theory.
\end{abstract}

\section{Keywords: Rotating Black Branes; Microscopic Configurations; State-space Geometry.}

PACS numbers: 04.70.-s Physics of black holes; 04.70.Bw Classical black holes; 04.70.Dy Quantum aspects of black holes, evaporation, thermodynamics.

*bellucci@lnf.infn.it

†bntiwari.iitk@gmail.com 


\section{Contents:}

1. Introduction.

2. $\mathcal{N}=1$ Supergravity Origin.

3. State-space Geometry.

4. Black Rings and Strings:

$4.1 \quad D_{1} D_{5} P$ Black Strings

4.2 Black Rings as Circular Strings

4.3 Small Black Rings.

4.4 Supertubes.

5. Conclusion and Discussion.

6. Future Directions and Open Issues.

\section{Introduction}

Recently, we have analyzed the state-space configurations of exactly fluctuating 1/2-BPS configuration 1] and subsequently extended the state-space analysis for a class of supersymmetric spherical horizon black holes [2, 3 . which preserve two real supersymmetries [4]. A set of interesting supergravity configurations [5] 7] provide further definite realizations for the present equilibrium statistical investigations. Several akin solutions also have been discovered in 5-dimensional gauged supergravity configurations [8 12. In particular, it has been realized since the study of BMPV black holes that the $D=5$ vacuum Einstein equations admit an asymptotically flat, stationary black hole solution with an event horizon of $S^{1} \times S^{2}$ topology which describes a rotating black ring [13. It turns out therefore that the state-space geometry of such a solution involves finitely many dissolved flux charges. The underlying argument lies on the fact that the ring solutions may not uniquely be determined by their conserved charges, mass and angular momentum, but it rather involves certain dipole charges. Moreover, we find that the asymptotic charges do not distinguish black rings from the black holes of spherical topology, and their intrinsic nature is intimately encoded in the state-space invariant objects. See for example, the solutions constructed in [14,15] describing an intriguing set of charged black rings. It thus appears natural to investigate the nature of state-space quantities of the horizon changing black hole solutions. We shall determine possible functional relations for the state-space configuration of black strings and black rings and compare concerned physical meanings of the global quantities with their spherical horizon counterparts.

Such a construction would clearly elucidate an important issue of the state-space geometry of the black rings of 14, 15], which entails the finite violation of the black hole uniqueness [13], since there are finitely many spacetime solutions with the same conserved physical charges. Physically, one finds that these configurations describe rotating loops of magnetically charged black strings. Since the loop is contractible, hence these solutions carry no net magnetic charge. However, these ring solutions do carry a non-zero dipole moment, which is a nonconserved quantity, often referred to as magnetic dipole charge, and thus we explore its plausible entanglement with underlying state-space geometry. In this case, the black rings of [16] correspond to the case of rotating circular strings, whose state-space geometry may easily be characterized by the mass, angular momenta, and a dipole charge. We find in general that the state-space geometric quantities thus obtained, in terms of a set of given conserved charge(s), dipole charge(s) and angular momenta, account for the underlying structure of the continuous infinity of five dimensional black brane configurations.

Furthermore, the dipole black ring charges have a simple microscopic interpretation [16], which may be obtained from the dimensional reduction of the eleven dimensional supergravity solution describing $M_{5}$-branes, with four worldvolume directions wrapped on an internal six torus and one worldvolume direction forming the $S^{1}$ of the black ring in the non compact dimensions. One thus finds that the dipole charge of black ring is nothing more than the number of $M_{5}$-branes present in the ring configuration. The most general solution of [16 has the three independent dipole charges, and thus describes a $(n+3)$ dimensional state-space manifold. The existence of such a configuration follows from the well known fact that the dipole charges arise from the orthogonal intersection of three stacks of $M_{5}$-branes wrapped on $T^{6}$, with the common strings of intersection forming the $S^{1}$ of the ring. Classically, the dipole charges contributing non-trivially to the state-space quantities are continuous parameters, whereas in the quantum theory they are quantified in terms of the number of branes in each stack. 
The conserved charges of supersymmetric black ring configurations may in fact be made arbitrarily close to those of the BMPV black hole by taking the small radius limit, but the ring cannot carry exactly the same conserved charges as that of the BMPV black hole. This is because dipole charges are required to specify the ring configuration, and subsequently entail the continuous violation of the four dimensional black hole uniqueness theorem [13 15. This article shows in characteristic that the state-space geometry of a ring configuration is relatively higher dimensional than usual BMPV black holes. Similar conclusions follow for the non-supersymmetric dipole rings which are prone to have definite phase transitions as well, and exactly in the same way as the supersymmetric black rings, they possess a relatively higher dimensional state-space manifold. However, if one takes charge quantizations into account, then this violation of uniqueness in both cases is rendered to be finite, see for details [17. The present consideration confronts with the case of very large charge configurations and treats the underlying brane charges as real variables.

Moreover, underlying angular momenta, along with the dipole momenta, play an important role in understanding the state-space geometry of yet an another class of higher dimensional black brane solutions. The recent solutions of supertubes [18 20] thus be our upright interest, whose analysis finds firm ground from the motivation of known state-space constructions [3. Here, the black ring configurations become black tubes, when lifted to higher dimensions. Ref. [15] identifies certain charged non-supersymmetric black tubes as thermally excited states of two-charge supertubes carrying $D_{1}$ and $D_{5}$-brane charges and a dipole charge associated to the Kaluza-Klein monopole (KKM), which correspondingly parameterize a three dimensional state-space geometry in the limiting picture of given rotation. Supertubes have further been the subject of an intense interest from yet a different direction, following the realization that the non-singular, horizon-free supergravity solutions describing tabular objects are thus in one to one correspondence with the Ramond sector ground states of the supersymmetric $D_{1}-D_{5}$ string intersections [21 23. In this regard, Mathur has conjectured that the supergravity solutions describing three-charge supertubes might similarly be accounted against the microstates of five dimensional supersymmetric black holes [24. Truthfully, Mathur's proposal has motivated a number of interesting studies for the $D_{1}-D_{5}$ systems and supertubes 25 32. Here, our construction of the state-space geometry examines their stability criterion, and concerns with an investigation of the statistical interactions which include the first supergravity example of non-singular horizon free three-charge supertubes of [25] 27 ].

The state-space investigations and the relationship among black rings, circular strings and supertubes 13 [15] may further be accomplished in the framework of supergravity solutions. Notice for instance that these configurations do not admit a supersymmetric limit with an event horizon, and this complicates the basic understanding of the microscopic origin of their entropy [16. However, there has been some progress made in this direction by studying a non-supersymmetric extremal ring with a non-vanishing horizon possessing leading order contribution. It has been conjectured, though, that the supersymmetric black rings should exist in this limit as well [28,29. Ipso facto, one may easily find that the conclusions drawn from our state-space computations are in perfect accordance with various additional ingredients of supersymmetry. The promising conclusions are important due to the following two reasons. Firstly, many of the well-known black ring solutions of [13 16] are classically believed to be unstable, whereas a supersymmetric black ring configuration should be stable. Secondly, it should be possible to facilitate a precise quantitative comparison between black rings, worldvolume supertubes, and the microscopic conformal field theory of the $D_{1}-D_{5}$ system.

We have thus been motivated to analyze these view-points from the perspective of their state-space geometry, and in particular focus our attention on the two-charge supertubes which have originally been discovered as the solutions of Dirac-Born-Infeld effective action [33] of an illustrious D-brane configuration in Minkowski vacuum [18. In the worldvolume picture, the branes associated to net charges are represented by the fluxes on the worldvolume of a tubular, higher-dimensional branes; while the latter description carries no net charge itself but only a dipole charge. In this description, the back-reactions on the space-time of branes have however been so far neglected [19,20. It appears from this analysis that the associated state-space geometry describes the statistical pair correlations, correlation volume, and in forthright, it indicates the nature of the phase transitions, if any, underlying these higher-dimensional supergravity configurations. The latter are thus prone to be affected by radiative corrections, viz., $\alpha^{\prime}$-corrections, multipole corrections, etc. Moreover, the present analysis signifies that the effects thus required, in order to procure the inconsistency between the two descriptions of the supertube configurations, might be viewed as refinements to the additional state-space co-ordinate, whose general transformations entangle with their intrinsic manifolds.

It is worth to mention that the state-space description has actually provided an extremely illuminating understanding for the physics of two-charge worldvolume supertubes. In turn, Ref. 30 32] have led to a new way of analyzing the counting entropy of the $D_{1}-D_{5}$ system, which does not utilize its CFT description. It is therefore desirable to have an analogous description for the three-charge supertube configurations, as well. 
However, the worldvolume description based on D-branes that incorporates the third dipole charge seems problematic, since it necessarily corresponds to an object which cannot be captured by the corresponding open string description, such as $N S_{5}$-branes or the Kaluza-Klein monopoles, see for instance [28 and references therein. Essentially, this difficulty can be circumvented by going to $M$-theory, where the three branes with net charges may be taken as three orthogonal $M_{2}$-branes, whereas the three dipoles are associated to three $M_{5}$-branes. This in turn is the most symmetric realization of the three charge supertubes, whose statistical configurations may thus easily be analyzed from the perspective of our state-space geometry.

Furthermore, Ref. [17] shows, in the lieu of the present investigation, that there exist concrete supersymmetric solutions, arising from the effective action of a single $M_{5}$-brane in the $M$-theory Minkowski vacuum [18, and that these configurations may at most carry up to four $M_{2}$-brane charges and six $M_{5}$-brane dipoles. In a generic set up, the state-space geometry turns out to be rather more involved and the effective supergravity solutions render to be calibrated supertubes, because the worldspace of $M_{5}$-branes takes the form $S \times C$, where $S$ is a calibrated surface and $C$ is an arbitrary curve living on the moduli space. Moreover, the three-charge calibrated supertube configurations capture all the dipoles, and one finds in this case that an arbitrary cross-section is possible for consistent supergravity solutions. It thus turns out, as expected from standard arguments orginating from [28, that these solutions in general suffer from certain limitations, and accordingly we notice that they capture only one of the angular momenta, as opposed to the two angular momenta present in the respective supergravity description. Here our goal is thus to provide a detailed discussion of the state-space geometry originating from the fundamental string theory and $M$-theory configurations and thereby to explore the related issues, which we shall present in the corresponding section of bubbled supertubes.

The present paper concentrates on the macroscopic-microscopic aspects of non-spherical configurations of consistent rotating black string/ ring solutions and discusses their state-space geometry, arising from the respective entropy obtained from the degeneracy of certain bound states, accounted either in terms of $D$-branes or $M$-branes. Therefore, the study of the equilibrium state-space geometry characterized by underlying electricmagnetic charges, dissolved fluxes, with or without angular momenta, is well suited for an important application of the AdS/CFT correspondence and determination of vacuum phase-transitions, if any, in the considered black brane configuration. This is because the state-space geometry has been one of the central intervention to explore the thermodynamic structures of large class of black holes and higher dimensional rotating black brane configurations [34 40]. See [41,42] for specific instances of various extremal and non-extremal branes, which have been comprising of the multi-centered branes, fractional branes, fuzzy rings and bubbling black branes. Ref. 3] exclusively has shown that the state-space geometry spanned by the set of consistent parameters is non-degenerate, regular and has a negative scalar curvature for the rotating Myers-Perry black holes, KaluzaKlein black holes, supersymmetric $A d S_{5}$ black holes, holographic corrected $D_{1}-D_{5}$ configurations and associated spherical horizon BMPV black holes in five space-time dimensions.

This approach indicates that contemplated configurations are effectively attractive and stable over a domain of an arbitrary hyper-surface of their state-space manifolds. It may nevertheless be noticed that there exists an intriguing relationship between non-ideal inter-brane statistical interactions and phase transitions. It may thus be observed that our state-space geometry analyzes the most probable interactions between the microstates of the black branes, and typically it determines the nature of statistical pair correlations, global correlation volume, phase transitions and certain other reasonable decay, if any, present in the underlying equilibrium statistical system. These premises may well be explored from the fundamental duality transformations on the charges which, in the large compactification radius limit, imply the existence of general coordinate transformations on an accompanying equilibrium state-space configuration. An important ingredient in the present discussion of state-space geometry thus follows from the fact that there exists a large class of rotating extremal black branes, non-extremal black branes, multi-centered black branes and the various other akin black brane solutions in string theory, $M$-theory and their generalizations which imply a set of interesting physical configurations. The statespace geometry is consequently expected to find an intriguing implication with the AdS/CFT correspondence, and its sound implication depends on an adequate account of the parameters defining the degeneracy of a large number of brane microstates associated with the rotating black strings/ rings.

This article has thus been organized into the following sections and their respective subsections depending upon the nature of underlying statistical fluctuations defining state-space configurations. The very first section provides concerned physical motivations to study the statistical fluctuations and thereby initiates why to study the concerned state-space geometry. Section 2 is devoted to a description of the origin of the state-space approach. In section 3, we briefly outline what is the state-space geometry, and analyze state-space pair correlation functions, which may be based on the parameters characterizing an ensemble of equilibrium microstates for the rotating black brane configuration under consideration. These specializations are substantially mimicked 
in 3, 41,42, which give covariant state-space geometric notions to accompanying statistical fluctuations about an equilibrium configuration. In section 4, we introduce the state-space geometry for the charged rotating black branes in string theory and $M$-theory. In particular, we shall focus our attention on the underlying state-space geometry of the various black strings and black rings. In this prominent section, we consider definite interesting limits of the supersymmetric black strings, and thereby examine the statistical correlations, which investigate the phase space stability and related features of underlying black brane configurations, in the view-points of string theory and $M$-theory. Here, we shall precisely divulge basic examples of three and four dimensional statespace manifolds which, as an intrinsic Riemannian geometry, may be defined from the negative Hessian of the entropy of black string and black ring configurations. Section 5 comprises final remarks and certain concluding notes of the state-space geometry being anticipated for the higher dimensional black strings/ rings arising from the string theory and $M$-theory compactifications. We shall then discuss the statistical implications associated with the boundary CFT brane microstates constituting the concerned configurations. Finally, we confront our construction of state-space manifolds with the other developments, related role of fluctuations in black brane configurations, and briefly address some open issues for future investigations.

\section{$2 \mathcal{N}=1$ Supergravity Origin}

In the present section we trace back the origin of the state-space approach, for non-spherical horizon black holes. The consideration of the state-space geometry may be described on the basis of a heuristic argument for predestined black rings and black strings, which exploits the possibility of higher dimensional black holes of non-spherical horizon topology. Candidly, the configurations of present interest may be obtained by taking five dimensional neutral black strings, constructed as the direct product of the Schwarzschild solution and a line with the horizon geometry $\mathbf{R} \times S^{2}$. A circular string may thus be obtained, if one imagines bending this string to form a circle, and so the topology now renders to be $S^{1} \times S^{2}$. It would thus be interesting to see how such topology changing effects are percieved from the perspective of our state-space geometry. In principle, the circular string thus described tends to contract by decreasing the radius of $S^{1}$, which follows simply, due to its tension and gravitational self-attraction. However, we can make the string to rotate along the $S^{1}$ and balance these forces against the centrifugal repulsion. Then we end up with a neutral rotating black ring, which as mentioned before, is a black hole with an event horizon of $S^{1} \times S^{2}$ topology.

Ref. [13] exhibits an explicit solution where such objects describe five dimensional vacuum general relativity configurations. This offers not only an example of non spherical horizon topology, but also turns out to be a counterexample to the ordinary four dimensional black hole uniqueness theorem. Originally, an investigation of rotating black ring solutions has given firm ground for an existence of vacuum black rings having rotation along the $S^{2}$ directions but no rotation along the $S^{1}$ ring circle 43 45]. In contrast to the foregoing rotating rings, the concerned authors have however found that the charged supersymmetric and non-supersymmetric black rings may be constructed in a more systematic way, and thus their state-space manifolds as well are an immediate particular of the present concern. We further wish to furnish the similar computations for the general black ring/ strings which may be defined as the $D$-dimensional black hole having $S^{1} \times S^{D-3}$ topology of (a spatial cross-section of) the event horizon. Correspondingly, we shall analyze the phase-space structures underlying in these configurations.

Let us now focus our attention on an intriguing limit of the state-space geometry associated with the wellknown supersymmetric black ring configurations. To be concrete, we would like to investigate the nature of state-space geometry of the five-dimensional supersymmetric black holes appearing in a subsector of $\mathcal{N}=1$ supergravity solutions. As mentioned earlier, these solutions are an example of asymptotically flat supersymmetric black hole of non-spherical horizon topology and consequently, it turns out that the underlying state-space geometry may be uniquely specified by its electric-magnetic charges and two independent angular momenta. The first example of our interest is simply associated with an asymptotically flat supersymmetric solution with regular event horizon having $S^{1} \times S^{2}$ topology. This in fact corresponds to the supersymmetric black ring configurations, whose existence have been conjectured in [28 and have further been physically supported by the investigations of [21,24.

It turns out that the state-space manifold of connoted non-spherical horizon solutions possess a richer structure than the ordinary BMPV spherical black holes, and in particular, it motivates us to investigate the nature of state-space configurations [3] for the general black rings and black strings in detail. The fact that the supersymmetry requirements impose no constraint on angular momenta of the ring solutions illustrates that the state-space geometry thus obtained may be co-ordinatized by the electric charges and two independent angular momenta of underlying equilibrium configuration. Moreover, it turns out that the solutions under consideration 
have a non-vanishing magnetic dipole, which may in an akin limit be fixed by the asymptotic charges, and therefore it is not an independent parameter of the concerned solutions signaling a lower dimensional statespace geometry. Although some black rings are believed to be unstable, it turns out that the supersymmetry requirement ensures that very solutions should be stable configurations. These notions may thus be appraised from the very incentive of our construction of respective state-space manifolds.

The novel feature of our state-space construction for the supersymmetric black ring solutions is associated with the fact that the angular momenta may be determined in terms of the dipole charges, conserved charges and compactification radius parameter $R_{0}$. It turns out that the radius $R_{0}$ may be determined as the function of $\left(J_{\psi}-J_{\phi}\right)$ and concerned dipoles. The removal of causal pathologies, such as closed causal curves necessitated in [46], consecutively initiates further analysis for the ring configurations, i.e. that the ring entropy must be a real valued function of the ring parameters, and thus the state-space configuration must be an intrinsic real Riemannian manifold. Nevertheless, it is known that the space-like section of the ring horizon implies that the horizon is geometrically a product of a finite radius circle $S^{1}$ and a two sphere $S^{2}$, and thus the ring entropy may easily be calculated from the horizon area as a function of the quantized charges and dipoles. As a matter of course, one may easily obtain the underlying state-space quantities and their statistical interpretation for the large charged supersymmetric black string and black ring configurations.

It is worth to note that the physical implication of the non-uniqueness theorem for the supersymmetric black rings and their statistical configurations force upon us that the problem of finding supersymmetric solutions may effectively be reduced to specifying appropriate sources as certain harmonic functions on the base space manifold [47. Physically, these sources describe $M_{2}$-branes with both worldvolume directions wrapped on the internal torus, and $M_{5}$-branes with four worldvolume directions wrapped on the torus: i.e. one finds the $M_{2^{-}}$ particles and $M_{5}$-strings living along the five noncompact directions. Moreover, the choice of the harmonic sources corresponds to a circular loop of $M_{5}$-strings with a constant density of $M_{2}$-particles distributed around the $M_{5}$-string. Therefore, it turns out that the parameters being involved directly lead to an appropriate set of co-ordinates on the state-space geometry which defines Gaussian fluctuations in the supersymmetric black ring solutions of [47].

What follows next shows that the state-space construction arising from the supertube configurations entails an admirable physical interpretation in terms of the parameters of underlying UV and IR CFTs. Specifically, the UV description indicates that the allied brane dynamics may be determined by the worldvolume theory which carries the conserved physical charges. In principle, the UV-CFT can also describe these black branes with a set of given charges for both the spherical and non-spherical horizon topology as different phases of the same theory, in which the dipole charges act as the parameters of the theory under consideration. Subsequently, one finds that the underlying theory has different IR flow, which depends upon the phase of UV-CFT. In fact, the spherical black hole phase renders to be exactly conformal, while the black rings induce a non-trivial flow to the IR-theory. It is nevertheless well known that the central charge of the IR theory is smaller than that of the UV theory [48, 49. This is because of the fact that the supersymmetry generators in one of the chiral sector of the $(4,4)$ UV theory are broken along the flow, and consequently the IR theory has only $(0,4)$ supersymmetry.

On other hand, the microscopic configuration of the black strings may be examined by considering the $D_{1} D_{5} P$ system whose parameters define state-space co-ordinates and thus the associated quantities. This connection is firm, due to the fact that the usual notion of the string theory implies that the microscopic description of black holes are typically based on the dynamics of brane configuration which has the same set of charges as that of the black hole. In this concern, an intriguing duality exists between the heterotic string and Type IIA superstrings, which controls the strong coupling dynamics of the heterotic string in diverse space-time dimensions [50]. In fact, an eleven-dimensional supergravity arises as a low energy limit of the ten-dimensional Type IIA superstring in the strong coupling dynamics of string theories. It is however worth to note in general that the black hole thus obtained may or may not have the ordinary spherical horizon topology. In fact, the black ring configurations can carry both the conserved charges and dipole charges: depending on which of the two sets one puts the stress on, their description is rather different. Interestingly, it turns out that the statespace geometry may in these descriptions be explored further, by investigating whether two intrinsic state-space manifolds are related by some general co-ordinate transformations. This is because the description based on conserved charges is the ultraviolet (UV) completion of the dipole based description, which in turn completely describes the physics of the string system at the lowest energies, that is the infrared (IR) description.

Both of these theories may however be described by usual two-dimensional sigma-models, and in the extremal limit where the momentum is chiral, the associated counting entropy formula follows simply from the Cardy formula which allows one to exhibit an intriguing state-space configuration. Note that the microscopic entropy of the rotating non-spherical horizon configurations may easily be obtained by using the central charge for the 
momentum available to distribute among the chiral oscillators, and thus the underlying state-space geometry would in principle differ in each description, even if the same object is referred. As mentioned before, it is the two-dimensional sigma-model, being regarded as an effective string, whose descriptions differ in what this string has been taken to be. It is interesting to notice that the IR theory has an effective string extending along the $S^{1}$ direction of ring, and thus the ring may be perceived as a circular string. The leading order analysis shows in particular that the invariant state-space global correlation scales as the inverse of the large charge counting entropy. For the extremal non-supersymmetric black rings of [16] and supersymmetric black rings of [48, 51, one finds that there exists an impressive match between the Bekenstein Hawking entropy and the corresponding statistical entropy which allows us to give a nice interpretation of the state-space geometry of typical five dimensional black brane configurations.

In order to investigate the possible state-space geometric perspective from the framework of UV theory [51], we may further consider an effective string which extends along the orthogonal dimension to the ring, and thus it follows that the concerned string configuration may be viewed as the supertube, or an excitation of it. Such configurations, which have been developed in [15] for two-charge black rings, and in [48] for the threecharge supersymmetric black rings, turn out to be quite interesting for our intrinsic geometric analysis. We may thus systematically examine the underlying state-space manifolds in each description and, in turn, a set of equilibrium parameters defines the associated virtues emanating from the state-space implications of the general three-charge higher dimensional rotating black brane configurations. One believes however that the pursuit of the space-time geometries may be capturing generic states of the CFT for chosen black holes and black rings with non-zero horizon area in leading and/ or subleading orders, and thus capturing enough of them to account for the horizon area of the macroscopic configurations. Here, we will use this result to be a crucial ingredient in the realization of the state-space fluctuations around equilibrium statistical configurations, for the higher dimensional rotating black string and black ring solutions.

As a next step towards this objective, it is instructive to explore the state-space geometry of black rings and relevant other implications in the limit of the circular string configurations. Distinguishably, we find that the view points of the IR theory ascribe an interesting picture to the underlying statistical correlation functions and related phase space properties over an equilibrium. This is because the worldvolume theories of black branes that carry a set of dipole charges have one of the worldvolume direction along the ring circle which describes the case of a circular strings, and thus may solely be based on the IR description of the underlying black ring configuration. The state-space geometry arising from the consideration of the microscopic theory turns out to be interesting, in the straight string limit of the circular rings of finite compactification radius. This arises from the fact that none of the proposed IR theories can distinguish between a ring and a Kaluza-Klein compactified string. Equivalently, the finite size radius corrections cancel out in the ring entropy, and thus in their state-space geometry.

It is thus akin to determine a convenient description of the state-space geometry for an extremal black ring with a finite horizon, whose entropy may be obtained in terms of the triple intersection of $M_{5}$-branes and a running momentum along the ring. Moreover, the concerned parameters of the solutions thus achieved determine the central charge of the corresponding theories, which describe the associated counting entropy and thus the state-space geometry of the ring solutions. Following Maldacena, Strominger and Witten (MSW), it turns out in characteristic that the central charge of the $(0,4)$ theory may be given by the number of moduli that parameterize the deformations of smooth intersections of concerned branes, and in turn is proportional to the number of branes of each kind [52. One may thus notice that the low energy dynamics can be described by a $(0,4)$-supersymmetric $(1+1)$-sigma model at the intersection of the branes, if the six compact directions of the internal compactifying space are small in the MSW consideration [52]. Furthermore, one finds from the IR description of such supergravity solutions in the underlying sigma-model framework that the present interest of the state-space geometry would be reasonable only when the volume of the six-dimensional internal manifold is far away from the respective value of the central charge of the microscopic theory.

An explication of the covariant state-space geometry for small black ring configuration is thus immediate. In order to do so, one may provide a string theory description to the structures required to accommodate different black objects, with the same conserved charges as that of a class of black rings under question. It turns out, once again, that the supertube configurations have the right topology to be identified as the microscopic description of small fluctuating circular configurations. Both of these configurations may simply be divulged as an ordinary intrinsic Riemannian manifold with non-vanishing global state-space correlations. Our analysis presumes nothing other than the supersymmetric rotating black rings with given two charges, and one dipole, and thus owing to the case of usual $K_{3} \times S^{1}$ compactifications. In this concern, the state-space configurations of two charged small black holes and related string theory and $M$-theory black brane solutions 41,42] show 
an akin connection with the non-spherical horizon black holes, what we shall analyze in the present paper. Furthermore, it turns out that the state-space geometry provides various useful informations in understanding the promising nature of underlying statistical configurations concerning small black holes, black strings, and black rings, in general. This may easily be justified from the fact that the underlying microscopic CFT assigns real finite entropy to the small fluctuating circular black rings. We may therefore identify that the state-space geometry describes definite fluctuations in the considered ensemble, and therefore, it renders an illuminating covariant representation, for the statistical pair correlation functions and the correlation volume, for the small black ring configurations.

We may thus anticipate the general consequences of our state-space investigations, which we shall illustrate for certain natural black hole/ string/ ring configurations. An important issue of the classical dynamical stability for generic black brane configurations turns out to be interesting in this regard, and as a result, the supersymmetry condition ensures that the supersymmetric black rings are stable upon quadratic fluctuations over the parameters defining the chosen configurations. Furthermore, one may easily realize that the nearsupersymmetric black ring configurations appear as well to have indisputably similar conclusions under the Gaussian fluctuation in the parameters which defines an underlying state-space geometry. Therefore, some qualitative and semi-quantitative arguments may be possible to make for the small range of the parameters of general higher dimensional solutions. In this picture, one may easily observe their global features from the associated scalar curvature of the state-space geometry. For example, there exist a black ring configuration, which branches at the cusp between the thin and fat black ring, and thereby the endeavored configuration must physically be an unstable black ring configuration. Similar arguments exist in the literature as well for the other black rings. In particular, by throwing any small amount of matter which adds mass but not angular momentum, we may easily see that there is no other black ring into which this system can evolve and thus it must back react violently $13,53,55$.

Most importantly, we may observe that such unstable branchings of the rings appear at the critical points of the concerned scalar curvature of the associated state-space manifolds defined in terms of the parameters of the ring configurations. Qualitatively, one expects that thin black rings suffer from the Gregory-Laflamme instability 56, which should grow lumps on the chosen ring, whose evolution may also be governed by the scalar curvature of the associated state-space manifold. Moreover, our intrinsic geometric study of the black rings and non-spherical horizon black holes exhibits that at least one unstable mode is added precisely at the state-space scalar curvature singularity, when going from thin to fat black rings. Thus, ring configurations may suffer from phase transition(s) and other vacuum instabilities, as well, under the general circumstances. Such a sort of instabilities which we have been considering here are indeed known in the literature and thus provide firm support for their concerned vindication. As a matter of course, these notions are rather well known for the fat black rings, i.e. that they should again be unstable [57. Note that the deductions thus made are further consistent with the known premise of the radial perturbations [55], against which all the fat black rings appear to be less stable than the thin black rings.

This alludes that the vacuum black rings with a single spin can be stable only if the Gregory-Laflamme instability are switched off and are far from the thin black rings with a small enough angular momentum. It may also be envisaged that the conclusions thus obtained for the non-spherical configurations may be generalized for the general vacuum black rings with two spins. In consequence, it is known that these higher spin rings also suffer from the similar superradiant instabilities with a rotating two-sphere [58. Having provided thus a brief account of higher dimensional black string/ ring configurations, one may note that there exists an asymptotic expansion of the exact spectrum of the degeneracy formula for large charges and angular momenta, which not only reproduces the entropy of the corresponding black brane solution in the leading order, but also to first few subleading orders as an expansion in the inverse power of the charges [59,60. Interestingly, the spectrum of half-BPS states in $\mathcal{N}=4$ supersymmetric string theory has adeptly been divulged [61, and thus one finds an appropriate notions of degeneracy among a set of correlated microstates. Given this correspondence between microscopic spectrum and macroscopic Bekestein Hawking Wald entropy of a black brane configuration, a natural question would be to understand a microscopic origin of the state-space geometric interactions arising from the degeneracy of the CFT microstates. In particular, what does this correspondence mean for the nonspherical horizon rotating black branes? This question is however beyond the scope of the present interest and thus would be relegated for future analysis. 


\section{State-space Geometry}

The present section provides a brief account of the state-space geometry, which as an intrinsic Riemannian manifold $(M, g)$ describes the nature of statistical fluctuations in the higher dimensional rotating black strings and rings. A similar analysis has recently been introduced in 3 for spherical horizon black holes, which we shall extend here for the black string and black ring solutions. We shall focus our attention on correlation functions and correlation volume of four parameter non-spherical horizon black branes, and thus study the nature of underlying statistical fluctuations. Besides many more standout references, the relevant details may be insinuated to 62 68 for basic introduction of thermodynamic state-space geometry; 34 39 for well-known black holes in general relativity; 40] for black holes in string theory; 41,42 for general state-space description of diverse pertinent black branes in string theory and $M$-theory; and [69] for the associated notions of chemical correlations and concerned relations with the quark number susceptibilities in 2 and 3-flavor Hot QCD configurations.

Following [3], it turns out in the entropy representation that the components of the covariant state-space metric tensor may be defined to be

$$
g_{i j}:=-\frac{\partial^{2} S(\vec{X})}{\partial X^{i} \partial X^{j}}
$$

It follows from our outset how the state-space geometry can be employed to describe fluctuating black ring/ string configurations. In order to do so, we shall restrict ourselves to the co-ordinates which are extensive parameters, and then draw local and global properties of the fluctuations about an underlying equilibrium statistical configurations. The variables $\left\{X^{i}\right\}$ shall be thought of, as a set of asymptotic parameters carried by the higher dimensional black hole and, in specific, they define a suitable co-ordinate chart on the state-space manifold. It is worth to mention that the coordinates $\vec{X}$ are a finite collection, consisting of the mass $M$, electric-magnetic charges $\left(P^{i}, Q_{i}\right)$, angular momenta $\left\{J_{i}\right\}$ and certain local charges $\left\{q_{i}\right\}$, if any. Thus, the most general co-ordinate, on an arbitrary intrinsic state-space manifold $(M, g)$, may be expressed as, $\vec{X}=$ $\left(M, P^{i}, Q_{i}, J_{i}, q_{i}\right) \in M$.

The case of two and three dimensional state-space manifolds have extensively been described in [3]. Here, we wish to explicitly offer the involved intrinsic geometric quantities for the four parameter black string/ ring configurations. Furthermore, we may also exhibit that the similar out-lines hold for a class of general multi-parameter black brane state-space configurations. For the black ring/ string configurations, the concerned four dimensional state-space geometry 11 may parametrically be defined by three possible parameters, viz., $\left\{X_{1}, X_{2}, X_{3}\right\}$, which shall be thought of, as the brane charges, momentum charges, or dipole charges; and an angular momentum $J$, which defines a well chosen sector of the microscopic theory. We may thus expose that the components of the covariant intrinsic metric tensor are given as

$$
\begin{aligned}
g_{X_{1} X_{1}} & =-\frac{\partial^{2} S}{\partial X_{1}^{2}} \\
g_{X_{1} X_{2}} & =-\frac{\partial^{2} S}{\partial X_{2} \partial X_{1}} \\
g_{X_{1} X_{3}} & =-\frac{\partial^{2} S}{\partial X_{3} \partial X_{1}} \\
g_{X_{1} J} & =-\frac{\partial^{2} S}{\partial X_{1} \partial J} \\
g_{X_{2} X_{2}} & =-\frac{\partial^{2} S}{\partial X_{2}^{2}} \\
g_{X_{2} X_{3}} & =-\frac{\partial^{2} S}{\partial X_{3} \partial X_{2}} \\
g_{X_{2} J} & =-\frac{\partial^{2} S}{\partial X_{2} \partial J} \\
g_{X_{3} X_{3}} & =-\frac{\partial^{2} S}{\partial X_{3}^{2}} \\
g_{J J} & =-\frac{\partial^{2} S}{\partial J^{2}}
\end{aligned}
$$

\footnotetext{
${ }^{1}$ In this section, we shall invariably use Euclidean notations for an intrinsic state-space manifold $\left(M_{4}, g\right)$, in the sense that the coordinates $X_{i}, X_{a} \in M_{4}$ (or corresponding notations $X^{i}, X^{a}$ ) follow contravariant tensor composition rules.
} 
Therefore, it is immediate to perceive that the components of the state-space metric tensor are related to respective statistical pair correlation functions, which may be defined in terms of the parameters describing the dual microscopic conformal field theory on the boundary. This is because the underlying metric tensor comprising Gaussian fluctuations in the entropy defines the state-space manifold of the rotating black brane configurations. Under this consideration, it is worth to note that the local stability of a fluctuating statistical configuration requires that the principle components of the state-space metric tensor, $\left\{g_{X_{a} X_{a}} \mid X_{a}=\left(X_{i}, J\right)\right\}$, which signify a set of heat capacities of the system, should be positive definite

$$
\begin{aligned}
g_{X_{i} X_{i}} & >0, i=1,2,3 \\
g_{J J} & >0
\end{aligned}
$$

The intrinsic geometric consideration shows that the state-space fluctuations between the two of the \{ branes, dipoles, angular momentum \} may be characterized as between two charges, between a charge and angular momentum, with pure angular momentum. These fluctuations are simply state-space second moments over the Gaussian distribution, which has been out-lined in 3. What has been shown for the spherical horizon black holes, we shall demonstrate here, i.e. that the similar conclusions hold for the state-space correlations for the non-spherical horizon black branes, as well. Specifically, the black strings and black rings show that the diagonal fluctuations involving a set of state-space co-ordinates are stable, or they rather weaken faster, and thus come relatively more swiftly in an equilibrium configuration, than those involving off diagonal state-space fluctuations.

Ref. [3] presents a detailed analysis of the relative state-space pair correlations, for the case of two charged rotating black brane configurations. As promised in the beginning of this section, in this paper we shall confront with four parameter black brane configurations. Thus, the desired three charged rotating black brane solutions can have the three brane charges and one rotations or three dipoles and one electric charge,..., etc. Under the present considerations, we may thus observe $\forall i \neq j \neq k \in\left\{X_{1}, X_{2}, X_{3}\right\}$ and, for an angular momentum $J$, that the relative state-space pair correlation functions form an intriguing qualification

$$
\begin{aligned}
C_{a b ; c d}:= & \left\{\frac{g_{i i}}{g_{j j}}, \frac{g_{i i}}{g_{i j}}, \frac{g_{i i}}{g_{i J}}, \frac{g_{i i}}{g_{j k}}, \frac{g_{i i}}{g_{j J}}, \frac{g_{i i}}{g_{J J}}, \frac{g_{i j}}{g_{i i}}, \frac{g_{i j}}{g_{k k}}, \frac{g_{i j}}{g_{J J}}, \frac{g_{i j}}{g_{j k}}, \frac{g_{i j}}{g_{j J}},\right. \\
& \left.\frac{g_{i j}}{g_{k J}}, \frac{g_{i J}}{g_{i i}}, \frac{g_{i J}}{g_{j j}}, \frac{g_{i J}}{g_{J J}}, \frac{g_{i J}}{g_{i j}}, \frac{g_{i J}}{g_{j k}}, \frac{g_{i J}}{g_{j J}}, \frac{g_{J J}}{g_{i i}}, \frac{g_{J J}}{g_{i j}}, \frac{g_{J J}}{g_{j J}}\right\}
\end{aligned}
$$

It should be noted in particular that the behavior of the set of independent relative state-space pair correlation functions is rather asymmetric, in comparison with the identical brane statistical pair correlations, or the other allowed relative pair correlations involving charge-charge and rotation-rotation components. This may nevertheless be physically understood, by the fact that the brane-brane interaction imparts relatively more energy, than that of the either existing state-space interactions and self-interactions. Moreover, we discover, with a non-trivial refinement, that the possible ratio of state-space pair correlations, without considering the respective inverse relative pair correlation functions, may be defined as

$$
\tilde{C}_{a b ; c d}:=\left\{\frac{g_{i i}}{g_{j j}}, \frac{g_{i i}}{g_{i j}}, \frac{g_{i i}}{g_{i J}}, \frac{g_{i i}}{g_{j k}}, \frac{g_{i i}}{g_{j J}}, \frac{g_{i i}}{g_{J J}} \frac{g_{i j}}{g_{j k}}, \frac{g_{i j}}{g_{j J}}, \frac{g_{i j}}{g_{k J}} \frac{g_{i J}}{g_{J J}}, \frac{g_{i J}}{g_{j J}}, \frac{g_{J J}}{g_{i j}}\right\}
$$

Following such a physically attractive classification, we may non-trivially deduce the nature of four parameter relative state-space pair correlations, and thus indicate the potential behavior of underlying black string/ ring fluctuating statistical configurations. This, as anticipated earlier, suggests whether equilibrium statistical systems are stable under the Gaussian fluctuations, or not, if either of the charges, dipole charges and angular momentum take certain specific values. Furthermore, we may inspect that there exists another choice involving two brane charges, one dipole and one angular momentum, which may also be easily carried out in the out-set of the present consideration.

These notions shall be further explicated in the next section for the specific black string/ ring/ supertube configurations, and we shall show that they pertain positive definite state-space correlations, if the two statespace co-ordinates take particular values. We shall in general analyze the following two interrogations:

1. For what value of the brane charge(s), the state-space (relative) pair correlations vanish?

2. Is there some relation of vanishing $f_{i j} \in \tilde{C}_{a b ; c d}$, with the vanishing entropy condition of a given black brane configuration?

For the state-space configurations of spherical horizon black holes [3, we know that the vanishing of distinct parameter state-space pair correlation functions happens exactly at twice the value of the vanishing entropy 
condition. We shall explicate these notions for non-spherical horizon black brane configurations and show in detail that their state-space geometry enjoys a lower bound, on the attainable brane charges or on the angular momentum, than the constraint arising from the acquainted large charged leading order black brane entropy solutions. In the next section, we shall show that the ratios of non-diagonal components vary as inversely, and in turn they remain comparable for a longer domain of the parameters defining Gaussian fluctuations in the entropy of specified black branes.

Moreover, we may see, from the positivity of the state-space metric tensor, that it imposes a set of stability conditions on the underlying statistical configuration, over the Gaussian fluctuations. It is however important to note that the metric conditions are not sufficient to insure global stability, for the chosen configuration, and thus one may, under this condition, only accomplish a locally equilibrium configuration. In fact, it is easy to express, in the present case, that the planar and hyper-planar stabilities of statistical configurations require that all the off-diagonal fluctuations must vanish. This implies that the principle minors $\left\{p_{2}, p_{3}\right\}$ of the underlying metric element should be strictly positive definite quantities on the entire state-space $\left(M_{4}, g\right)$. An elementary analysis shows that the principle minors may be represented as the following determinant of the components of the metric tensor:

$$
\begin{aligned}
& p_{2}:=\left|\begin{array}{ll}
g_{11} & g_{12} \\
g_{12} & g_{22}
\end{array}\right|>0, \\
& p_{3}:=\left|\begin{array}{lll}
g_{11} & g_{12} & g_{13} \\
g_{12} & g_{22} & g_{23} \\
g_{13} & g_{23} & g_{33}
\end{array}\right|>0
\end{aligned}
$$

An existence of positivity of the state-space volume form imposes a stability condition on the Gaussian fluctuations, over underlying equilibrium statistical configurations, i.e. that one must have $p_{4}:=\|g\|>0$. This naturally requires that the determinant of the state-space metric tensor must be a positive definite function of the electric-magnetic charges, fluxes, if any, and angular momentum. A straightforward computation thus yields that the determinant conditions of the metric tensor may be given as

$$
\|g\|:=\left|\begin{array}{llll}
g_{11} & g_{12} & g_{13} & g_{1 J} \\
g_{12} & g_{22} & g_{23} & g_{2 J} \\
g_{13} & g_{23} & g_{33} & g_{3 J} \\
g_{1 J} & g_{2 J} & g_{3 J} & g_{J J}
\end{array}\right|>0
$$

This precisely entails that the state-space determinant and hyper-determinant of the metric tensor must be positive definite. Thus, the complete stability condition of a black string/ ring configuration requires that the principle components of the Gaussian fluctuations should be positive definite and the other components of the fluctuations must vanish 64. In order to ensure this condition, we may appraise that all the principle minors of the concerned state-space metric tensor must be a strictly positive definite quantity for the chosen sector of the system. The global stability condition thus forces us that the set of simultaneous equations expressed by the principle minors must satisfy positivity, in the domain of interest of the parameters of black hole configuration. In other words, the principle minors constraints, viz. $\left\{p_{i}>0 \forall i=1,2,3,4\right\}$, should hold under the consideration.

It is worth to note that the determinant condition $\|g\|>0$ implies that there exists a positive definite volume form on $\left(M_{4}, g\right)$. However, in this case, one finds that the classical thermodynamic fluctuation theory may not remain viable, for the general black string/ ring configurations, see for further details 3. This is because a black brane cannot come into an equilibrium with any extensive, infinite environment. Thus, the full Hessian determinant of the black brane entropy $S:=S\left(X^{i}\right)$ turns out to be negative definite, when all the asymptotic parameters are fluctuating, see for a motivation 64, 70. This is also required, in order to produce a local maximum of the total entropy of the chosen configuration. Thus, the non-existence of a positive definite volume form on the intrinsic state-space manifold $\left(M_{4}, g\right)$ puts the full problem beyond the control of standard equilibrium fluctuation theory, when all the state-space co-ordinates $\left\{X^{i}, J\right\}$ are fluctuating.

Physically, one may justify it, by saying that the brane dynamics ultimately favors extreme situations, where the black brane configuration may either completely evaporate, or grow without limit. In fact, the black brane will presumably create whatever particles or branes it likes near its event horizon, and an equilibrium is thus quite unlikely, until the environment is populated by the particles or branes, in the same proportion to those which have been created. Nonetheless, in a limited domain of the parameters, when at least one of the parameters is slowly changing in time, with respect to the remaining ones, obliges a class of stable configurations 
under the Gaussian fluctuations. This investigation implies a class of stable configurations with $n$ number of fluctuating parameters, and the remaining $(4-n)$ parameters being effectively fixed, or drifting very slowly out of the equilibrium configuration with the environment, where $1 \leq n \leq 4$.

Furthermore, one may note that the scalar curvature corresponding to this state-space geometry elucidates the typical feature of Gaussian fluctuations about an equilibrium brane microstates of the desired rotating string/ ring configurations. Furthermore, we may easily procure that the scalar curvature determines a global invariant on the four dimensional state-space manifold. In this case, it thus follows that one may explicate the average nature of underlying microscopic black brane configurations. Such an interesting invariant, which accompanies the information of global correlation volume of underlying statistical systems, turns out to be the intrinsic state-space scalar curvature. A detailed analysis shows that the general form of the concerned scalar curvature may be expressed as

$$
R=\frac{1}{2\|g\|^{3}} N\left(X^{i}, J\right)
$$

The exact expression for the numerator $N\left(X^{i}, J\right)$ is rather involved and thus we relegate it for the specific considerations. Consequently, it may be noticed that a systematic examination demonstrates that the statespace geometry with a higher number of charges and angular momenta are similarly not unlikely to define. In this concern, we remark that one may easily deduce the statistical correlations and stability criteria, in terms of the corresponding parameters of an ensemble of microstates, which characterize a possible elementary statistical basis against the macroscopic black brane configurations.

Thence, one may envisage, from the theory of the Gaussian distribution, that the components of the covariant state-space metric tensor may always be defined as the negative Hessian matrix of the underlying entropy with respect to the mass, invariant charges, and angular momenta, as well as possible exotic fluxes, if any, carried by the black brane configuration. It is noteworthy, in the present case, that the black rings and black strings, when viewed from out-side, may only be characterized by their mass, angular momenta, and concerned asymptotic charges. Thus, the detailed internal structure and history of their formations are rather irrelevant, to an asymptotic observer, within the validity limit of the "no hair" property. Such a drastic reduction of complexity is clearly an important characteristic of our state-space investigation for the black string/ ring configurations, whose spherical horizon case have been considered in [3].

Ruppenier has also interpreted, with the assumption "that all the statistical degrees of freedom of a black hole live on the black hole event horizon", that the scalar curvature signifies the average number of correlated Planck areas on the event horizon of the black brane [71. Essentially, the zero scalar curvature indicates certain bits of information on the event horizon fluctuating independently of each other, while the divergent scalar curvature signals some phase transitions indicating highly correlated pixels of the informations. Moreover, Bekenstein has conjointly introduced an elegant picture for the quantization of the area of the event horizon, being defined as an integral multiple Planck area [72. The concerned examination thus signifies that the present analysis has a microscopic resolution that rather involves the framework of well-celebrated Mathurs fuzzballs [73].

Our geometric perspective thus discloses an appropriate ground, with the statements that the state-space scalar curvature of interest has not only exiguous microscopic knowledge of the black hole configurations, but also it has an intriguing intrinsic geometric structure. Specifically, we expose that the configurations under present analysis are effectively attractive or repulsive in general, while they are stable, only if at least one of the parameter remains fixed. Finding statistical mechanical models with like behavior might yield further insight into the microscopic properties of black branes. Thus, the hope is that our state-space analysis would provide a set of conclusive physical interpretation encoded in the scalar curvature and related intrinsic geometric invariants, for the black stings/ ring solutions.

Incrementally, there exists a series of interesting circumstances, which support the fact that our study offers a geometrical approach to analyze the microscopic configuration of an ensemble of microstates, which describe an equilibrium thermodynamic configuration, and thus one may actually apply it to divulge important physical and chemical behavior of the string theory/ $M$-theory black brane configurations. In this paper, we specifically wish to consider a general situation of the higher dimensional non-spherical horizon black hole configurations, where the concerned state-space geometry determines the statistical pair correlation functions and correlation volume, and thus it may disclose a certain critical nature of the associated dual boundary conformal field theory.

Thus, the distinguishing features of microscopic acquisitions, which are well-complied with an understanding of the associated parameters defining the limiting macroscopic configuration, may easily be exhibited. In fact, it is known that both the apprised descriptions may further be elucidated from the very likely application of the AdS/CFT correspondence and LLM geometries [23. General intrinsic geometric appreciation may be envisaged via the associated degeneracy formula of the black brane microstates, which we shall relegate to other 
future investigations. The present consideration nevertheless finds that the state-space scalar curvatures, as a function of the parameters, entails the central nature of the stabilities of the considered black strings/ rings, while the corresponding determinant of the state-space geometry, as analyzed earlier, when showing that the most probable states are far apart, sheds light upon the notion of the degeneracy of an ensemble of brane microstates constituting novel equilibrium statistical configurations.

\section{Black Rings and Strings}

The present section considers intrinsic geometric aspects of the rotating higher dimensional black branes and analyzes associated state-space configurations for the case of charged and dipole charged rotating black strings/ rings. In order to see this point more clearly, we shall focus our attention on the number of branes, which define the charges associated with five dimensional black string and black ring configurations. Our study relies on the $S O(4)$ rotation group in $(4+1)$-dimensions, which contains two mutually commuting $U(1)$ subgroups. This means that it is not unlikely to have rotations in two independent rotation planes, which thus generate two independent angular momenta $J_{\psi}$ and $J_{\phi}$. As often the case in General Relativity, it would indeed be convenient here too to work with the adapted state-space coordinates, which are possible parameters of the space-time solutions under question.

To be concrete, we shall specifically consider the ring space-time extending along an insistent spatial plane, rotating around the $\psi$ direction. Thus, we may easily describe the non-vanishing contribution of the angular momentum $J_{\psi}$ into the concerned state-space geometry. Nevertheless, our general idea would be to find the state-space properties of higher dimensional black brane configurations. We begin by constructing a foliation of flat space, in terms of the equipotential surfaces of the field created by a source term, resembling the black string/ ring one is seeking. For instance, Ref. [74] considers an approach to obtain state-space coordinates suitable for black hole configurations and those of the similar black strings on a Kaluza-Klein circle. Instead of considering the equipotential surfaces of a scalar field sourced by a ring, it is sometime more convenient to work directly with the equipotential surfaces of an associated two form potential: $B_{\mu \nu}$. It turns out that one may regard the ring as a circular string, which in an underlying effective field theory description acts as the electric source of the three form field strength $H=d B$.

For example, it is not difficult to construct the solution associated with two form fields, for the circular electric source of a ring, having Kaluza-Klein radius $R_{K K}$ and phase between 0 and $2 \pi$ (by using the familiar methods from classical electrodynamics, see [19]). In fact, one finds, in five space-time dimensions, that the electric-magnetic dual of the field $H$ satisfies

$$
* H=F=d A \text {, }
$$

where $A$ is the standard one-form gauge potential. The dual of the electric string is in fact a magnetic monopole, which here is just a circular distribution of the monopoles, whose analysis we shall furnish in the subsection 3.2. Note further that the space-time invariant/ asymptotic parameters being adapted for the two form potential $B$, also facilitate greatly the correct choice of the co-ordinate charts for their state-space manifolds. We shall thus analyze the state-space configurations arising fom the solutions with gauge dipoles, which have been of definite interest for the case of supersymmetric black rings. For the purpose of understanding three-charge space-time geometries dual to the microstates of $D_{1}-D_{5}-P$ CFT, one is essentially not so much interested in the black rings with a regular event horizon itself, but rather in the zero-entropy limit of the rings, which for simplicity we shall refer to, as the three charge supertubes.

Motivated from 28, 29, we shall confront with the supersymmetric black rings, whose existence have also been based on thought experiments involving supersymmetric black holes and bubbled black branes, and show almost everywhere that they find a non-degenerate state-space geometry, which has a non-vanishing scalar curvature, and thus corresponds to an interacting statistical system. The subsequent discovery to classify supersymmetric solutions of five-dimensional $\mathcal{N}=1$ supergravity also facilitates several state-space configurations involving supersymmetric black rings, charged black rings and other related black brane solutions, see for instance [15, 75. Thus, the necessary and sufficient condition for the solutions being supersymmetric is that they reduce to a class of simple four-dimensional base space manifolds, such that there exists a canonical form for specific non-spherical horizon black brane solutions 9, 46. In this case again, the concerned state-space geometry turns out to be generically well-defined, and corresponds to interacting statistical configurations. In order to systematically present our analysis, we shall relegate the detailed investigations of these solutions to the final subsection 4.4 . 
In order to take a closer look at generic black brane microscopic configurations, one may consider an intriguing idea of bubbling supertubes and foaming black holes, what Bena and Warner have appraised in [76. In particular, they have constructed smooth three-charge BPS geometries, which resolve space-time singularity of the zero entropy $U(1) \times U(1)$ invariant black ring configurations. The concerned singularity may be resolved by geometric transitions, which result into the geometries without branes sources or singularities, but the daughter solution arises with non-trivial horizon topology. The underlying geometries are both ground states of the black ring, and non-trivial microstates of the $D_{1}-D_{5}-P$ system. Thus, one finds the form of the space-time geometries, which result from the geometric transition of the $N$ zero-entropy bubbled black ring solutions. Furthermore, coincident general geometries, being parameterized by $6 N$ functions, give rise to a very large number of smooth bound-state three-charge solutions, and thus have $6 N$ dimensional state-space configurations. The generic microstate solutions turn out to be specified by the four-dimensional hyper-Kähler geometry of chosen signature, and contain the foam of non-trivial two-spheres. In turn, there exist definite conjectures that these geometries account for a significant part of the entropy of the $D_{1}-D_{5}-P$ black holes, and thus the Mathur's conjecture 73 . may be reduced to counting certain four-dimensional hyper-Kähler manifolds.

A general three-charge supertube solution is however given by six arbitrary functions: four of them determine the shape of the object, three describe the charge density profiles, but the functional constraint coming from setting to zero the event horizon area makes the choice appropriate 47,77. The near-tube geometry is of the form $A d S_{3} \times S^{2}$ and, since the size of the $S^{2}$ is finite, the space-time curvature is everywhere low. However, since the $\mathrm{AdS}_{3}$ is periodic around the ring, these solutions in fact have a null orbifold singularity, whose statespace configurations may elsewhere be likewise analyzed. In order to obtain smooth, physical space-time and state-space geometries corresponding to supertubes given by six arbitrary functions, one must learn how to resolve the space-time orbifold singularity. Thus, what are its consequences for the concerned state-space coordinates remains an intriguing question. Nevertheless, we wish to relegate these issues at this point and would like to furnish them at appropriate receptacles. The specific state-space configurations shall now systematically be divulged for promising black strings and black rings, and further details are presented in their respective subsections. Finally, the section 4 offers a set of definite conclusions, and section 5 out-lines encouraging remarks for the future.

\section{1 $D_{1} D_{5} P$ Black Strings}

In this subsection, we shall initiate our state-space geometric investigation for the simplest rotating non-spherical horizon solution and compute the underlying quantities, such as the curvature scalar for the rotating black string configurations for a given KaluzaKlein momentum. We shall also require that the concerned electric-magnetic charges, angular momentum, and dipole charges, if any, of the solution have a fixed symmetry group, and thereby focus our particular attention on the specific sector of the dual $D_{1}-D_{5}-P$ CFT describing three charge black brane configurations with unit Kaluza-Klein momentum.

Most of the progresses in understanding whether the $D_{1}-D_{5}-P$ microstates are dual to the bulk solutions have occurred on two apparently distinct fronts. The present paper thus investigates the intended behavior of underlying thermodynamics and statistical mechanics in the perspective of state-space geometry, and expresses the concerned quantities in terms of the parameters of bulk/ boundary configurations. In fact, we shall make clear that the first case may be involved in finding two point state-space correlation functions defined via the parameters of individual smooth solutions carrying brane charges. One thence may go for analyzing the global properties of the underlying state-space manifold, which may be obtained in terms of the parameters of the microscopic CFT 25, 27,78,79. The detailed construction of the $D_{1}-D_{5}-P$ configurations nevertheless confirms that some CFT microstates, which are dual to the bulk geometries, have an interesting intrinsic statistical feature, and in fact it seems that the nature of the equilibrium configuration may be highlighted from the out-set of the state-space geometry.

We shall secondly be involved in understanding the stability of rotating brane configurations, i.e. a physics issue behind the statistical existence of these CFT solutions, and thus would be interested in analyzing the simplest definite string theory and $M$-theory configurations from the perspective of the state-space fluctuations. It has remarkably been shown in 28] that there exists a very large class of black brane configurations, with three charges and three dipole charges, which can have an arbitrary shape, and consequently generalize the twocharge supertube considerations [80]. This is because the entropy of the $D_{1}-D_{5}$-system comes from arbitrary shapes of the two-charge supertubes, and thus it is natural to wonder how the arbitrary shapes of three-charge supertubes account for the sizable part of counting the entropy of $D_{1}-D_{5}-P$ black holes. As a matter of course, we shall first investigate what is the nature of underlying state-space manifolds for the $D_{1}-D_{5}-P$ black string 
configurations, with a given Kaluza-Klein momentum charge, and subsequently explore the consideration for general momentum excitations.

In what follows next, in order to make tangible contact of our state-space geometry with the microscopic perspective of rotating black objects, we shall consider an enticing case of black string solution whose microstates may be defined via the parameters of $D_{1}-D_{5}-\mathrm{CFT}$. It is nevertheless known, for unit Kaluza-Klein momentum, that the counting entropy of deliberated black string is given by

$$
S\left(Q_{1}, Q_{5}, J\right):=\pi \sqrt{Q_{1} Q_{5}-J^{2}}
$$

A simple calculation thence finds that the components of the covariant metric tensor, defined as the negative Hessian matrix of the string entropy, with respect to its $D_{1}, D_{5}$-brane charges and angular momentum $J$, can be expressed as

$$
\begin{aligned}
g_{Q_{1} Q_{1}} & =\frac{\pi}{2} Q_{5}^{2}\left(Q_{1} Q_{5}-J^{2}\right)^{-3 / 2} \\
g_{Q_{1} Q_{5}} & =-\frac{\pi}{2}\left(Q_{1} Q_{5}-2 J^{2}\right)\left(Q_{1} Q_{5}-J^{2}\right)^{-3 / 2} \\
g_{Q_{1} J} & =-\pi J Q_{5}\left(Q_{1} Q_{5}-J^{2}\right)^{-3 / 2} \\
g_{Q_{5} Q_{5}} & =\frac{\pi}{2} Q_{1}^{2}\left(Q_{1} Q_{5}-J^{2}\right)^{-3 / 2} \\
g_{Q_{5} J} & =-\pi J Q_{1}\left(Q_{1} Q_{5}-J^{2}\right)^{-3 / 2} \\
g_{J J} & =2 \pi Q_{1} Q_{5}\left(Q_{1} Q_{5}-J^{2}\right)^{-3 / 2}
\end{aligned}
$$

Physically, the principle components of the state-space metric tensor signify heat capacities or the relevant compressibilities, whose positivity connotes that the underlying system is in locally stable equilibrium configurations of the $D_{1}$ and $D_{5}$-brane microstates. We may thus observe that the state-space geometry, materializing from the Bekenstein-Hawking entropy of the $D_{1}-D_{5}$ black string configurations, admits remarkably simple expressions in terms of the brane charges and angular momentum. It may thence be suggested that the plausible microscopic preliminaries might be expected from the state-space configuration, which in the real sense would be obtained from the Cardy formula or associated general Hardy-Ramanujan formula. As enumerated in the previous section, one can specifically appreciate the positivity of the principle components of the present state-space metric tensor, and thus find, for all non-zero brane charges, viz., $Q_{1}, Q_{5}$ and angular momentum $J$, that they comply

$$
\begin{aligned}
g_{Q_{1} Q_{1}} & >0 \\
g_{Q_{5} Q_{5}} & >0 \\
g_{J J} & >0
\end{aligned}
$$

Furthermore, it may easily be seen that the ratios of diagonal components vary as the inverse square of the underlying parameters which change under the Gaussian fluctuations, whereas the ratios involving off diagonal components vary only inversely, in the chosen domain of parameters of equilibrium black string configurations. This suggests that the diagonal components weaken faster and relatively more quickly come into an equilibrium, than the off diagonal components, which remain comparable for the longer domain of associated parameters defining the $D_{1}-D_{5}$ configurations. Importantly, we may easily substantiate, for the distinct $i, j \in\{1,5\}$, that the relative pair correlation functions satisfy

$$
\begin{aligned}
\frac{g_{Q_{i} Q_{i}}}{g_{Q_{j} Q_{j}}} & =\left(\frac{Q_{j}}{Q_{i}}\right)^{2} \\
\frac{g_{Q_{i} Q_{i}}}{g_{J J}} & =\frac{Q_{j}}{4 Q_{i}} \\
\frac{g_{Q_{i} J}}{g_{Q_{j} J}} & =\frac{Q_{j}}{Q_{i}} \\
\frac{g_{Q_{i} Q_{i}}}{g_{Q_{i} J}} & =-\frac{Q_{j}}{2 J} \\
\frac{g_{Q_{i} J}}{g_{J J}} & =-\frac{J}{2 Q_{i}} \\
\frac{g_{Q_{i} Q_{i}}}{g_{Q_{j} J}} & =-\frac{Q_{j}^{2}}{2 J Q_{i}}
\end{aligned}
$$


Moreover, it is intriguing to notice that the behavior of the brane-brane statistical pair correlation function, which is defined as $g_{Q_{1} Q_{5}}$ is rather asymmetric in contrast to the other existing pair correlation functions. In fact, one may understand it, by arguing that the brane-brane interactions impart more energy than the other captivated state-space interactions, viz., either corresponding to the self-interactions or that of the rotations. The present analysis thus proclaims that the relative pair correlation between the $D_{1}-D_{5}$ branes, with respect to rotation-rotation correlations, can be expressed as

$$
\frac{g_{Q_{1} Q_{5}}}{g_{J J}}=-\frac{1}{4}\left(1-\frac{2 J^{2}}{Q_{1} Q_{5}}\right)
$$

This implies that the relative brane-brane statistical pair correlation function vanishes exactly at the half value of the angular momentum, compared to that of the vanishing black string entropy condition. It may further be explicitly seen, from the non-vanishing central charge, that the brane-brane statistical pair correlation is stable, only if the underlying angular momentum satisfies a lower bound $|J|<\sqrt{\frac{Q_{1} Q_{5}}{2}}$. Similarly, one may contemplate the nature of other relative brane-brane statistical pair correlation functions, as well, and in turn, we find that the ratios, such as $g_{Q_{i} Q_{j}} / g_{Q_{i} Q_{i}}$ and $g_{Q_{i} Q_{j}} / g_{Q_{i} J}$ may easily be analyzed for all $i, j \in\left\{Q_{1}, Q_{5}\right\}$.

For to investigate the global properties of fluctuating $D_{1}-D_{5}$ configurations, we need to determine the stability along each intrinsic direction, each intrinsic plane, as well as on the full intrinsic state-space manifold. Intimately, to determine whether the underlying configuration is (locally) stable on state-space planes, one may thence compute the corresponding principle minors of the negative Hessian matrix of the black string entropy. In this case, we may easily appraise that the set of principle minors $p_{i}$, computed from the above state-space metric tensor, reduces to

$$
\begin{aligned}
& p_{0}=1 \\
& p_{1}=\frac{\pi}{2} Q_{5}^{2}\left(Q_{1} Q_{5}-J^{2}\right)^{-3 / 2} \\
& p_{2}=\pi^{2} J^{2}\left(Q_{1} Q_{5}-J^{2}\right)^{-2}
\end{aligned}
$$

We thus see that the hyper-planar state-space configurations are stable and consequently the $D_{1}-D_{5}$ black string configuration remains stable under the Gaussian fluctuation, if at least one of the parameter is held fixed. Notice further that the determinant of the metric tensor $\left(\|g\|=p_{4}\right)$ is identically zero for the exemplified black string entropy calculation, which microscopically holds as an asymptotic expansion of the solution, in the limit of large brane charges. We may nevertheless see that the constant entropy curve, which defines the degenerate state-space configuration, may be given as

$$
Q_{1} Q_{5}-J^{2}=c
$$

where the real constant $c$ may, for a given entropy $S_{0}$, be defined as $c:=\frac{S_{0}^{2}}{\pi^{2}}$.

In order to extensively appreciate the present consideration, it would further be instructive to extend our state-space analysis to the most general three charge rotating configurations. In this concern, the $D_{1}-D_{5}-P$ black branes exhibit that the state-space manifolds associated with BPS black string/ ring solutions have two microscopic interpretations: one in terms of the parameters of the $D_{1}-D_{5}-\mathrm{P}$ CFT [48, and another in terms of the parameters of the four dimensional black hole CFT [48,51,81]. This is because the rings on the first hand are described via the parameters of the black ring CFT, and thus they should be thought out as those of the ground states of the BPS black ring microstates, in the same way as the parameters of the solutions of [82,83] arising from the ground state of five-dimensional three charge black holes, and the other set of solutions of [84, yielding the ground state of the four dimensional four charge black holes, which are dual to the vacua of $D_{1}-D_{5}$-P CFT. Nevertheless, the present analysis remains independent of the CFT vacua, and the concerned intrinsic statespace manifolds correspond to Gaussian fluctuations over the equilibrium configurations. At this point, an exact establishment of underlying state-space correlations with the CFT relations is left for a future investigation.

Based on the microscopic description of supertubes [48, 82, 83, it may however be anticipated that our geometric construction would correspond to multiple supertubes, which are dual to CFT states, with longer effective strings than the solutions that come from only one supertube. Hence, the maximum correlation length in the set up of our intrinsic state-space manifolds, in the limit of largest number of brane bubbles, should correspond to an ensemble of CFT states with the longest effective strings, which are the ones that give rise to the notion of effective state-space correlations for the $D_{1}-D_{5}-\mathrm{P}$ black brane configurations. It is worth to mention that the state-space geometry stems naturally from the implementation of the machinery of intrinsic 
Riemannian geometric Gaussian fluctuations to the most general rotating black brane configurations. Moreover, the underlying physics in a chosen microscopic sector seems closely related to that of the other systems containing the correct tower of black hole/ ring/ supertube ground states.

As mentioned earlier, the BPS black rings have two microscopic interpretations: one in terms of $D_{1}-D_{5}-\mathrm{P}$ CFT [48, and the other in terms of the four dimensional black hole CFT 48, 51, 81. Hence, our constructions should similarly regarded, so that both the microstates of the $D_{1}-D_{5}-P$ system, as well as those of the four dimensional black hole CFT, indistinguishably describe the state-space of concerned black rings or generic black branes. The results thus obtained indicate a number of interesting consequences and promising suggestions for future work. An important exert would consequently be to start from a given $D_{1}-D_{5}-P$ microscopic description of the black ring solutions, and to explore them in the zero-entropy limit. In turn, our analysis also indicates that the bound states, when the brane could be determined by a gas of positive and negative centers, with fluxes threading the many non-trivial two-cycles of the Gibbons Hawking base, and having no localized brane charges on them, would thence characterize interesting state-space manifolds. This result adheres an appropriate understanding of the generic horizon changing black brane configurations.

Interesting features of the state-space covariant objects associated with supersymmetric black strings/ black rings have been based on the fact that these solutions may uniquely be specified by their electric charges, dipole charges, if any, and angular momenta, which, in the limit of vanishing compactification radius, render the same conclusion, i.e. that these solutions reducing to spherical horizon BMPV black hole ones would in general have non-vanishing state-space pair correlation functions [3]. In fact, there are no black ring solutions with $J_{\psi}=J_{\phi}$, and thus the co-ordinate charts of the corresponding state-space geometry, as a collection of the parameters of concerned spherical horizon black brane configurations, would always be different from those of the conserved charges of the BMPV black holes.

At this point, it may be observed that the entropy, as a function of the parameters of black ring solutions, is rather maximized in the zero radius limit [28, and thus the geometric objects, as the function of angular momenta for fixed and equal brane charges, viz., $Q=Q_{i}$, may be obtained from the degeneracy which is not continuous in this domain. As we shall see in the coming subsections, this observation may easily be justified from the reduction of ring solutions to the BMPV black holes, whose entropy is always greater than the entropy of the limiting zero radius black ring configurations. As a consequence, there always exists a discontinuity, which is due to the change in horizon topology from $S^{1} \times S^{2}$ at positive radius to $S^{3}$ at zero Kaluza-Klein compactification radius. Analogous geometric observations may thus be entertained to the discontinuous increase in their entropy, i.e. that the continuity occurs for the multi-centre extremal Reissner-Nordström black holes, when the two sources become coincident. Thus, the reduction of the dimensions of concerned state-space manifold, which is the matter of the next subsections, is quite natural.

\subsection{Black Rings as Circular Strings}

In order to describe the state-space geometric origin of black ring solution, we may focus on the corresponding conserved charges adherinf to $M_{2}$-branes, which can be obtained by turning the worldvolume fluxes on. In particular, if we set the worldvolume fluxes off, then the ring carries only $M_{5}$ dipoles and angular momentum $J_{\psi}$, which in the absence of fluxes may be identified with an effective string momentum that defines the central charge of the corresponding aforementioned IR theory. Although this configuration, at any finite radius, turns out to be non-supersymmetric, even if the momentum is chiral, however the extremal limit of the dipole ring solution corresponds to the MSW black branes [52. The present subsection is thus devoted to analyze an associated state-space behavior of leading order dipoles black ring/ string configurations.

Intriguingly, the world volume theory of branes carrying definite dipole charges, in which the world volume direction is along the direction of the ring circle, describes an effective extended string which may be considered as the circular string, after carrying out the associated Kaluza-Klein compactification. Such a description of the extremal black ring with definite horizon area may be obtained in terms of the triple intersection of $M_{5^{-}}$ branes, with momentum running along the ring direction. In turn, the concerned low energy dynamics may be described by the $(0,4)$-supersymmetric $(1+1)$-sigma model at the intersection of underlying branes. To be specific, let us focus our attention on the charges that correspond to the case when the fluxes on the $M_{2}$-branes are turned off, and only $M_{5}$-dipoles fluctuate. In this case, the ring state-space configuration can by divulged by only the $M_{5}$-dipole charges $\left\{n_{1}, n_{2}, n_{3}\right\}$ and angular momentum $J_{\psi}$. It is worth to note, in this case, that the ring solution, in turn, does not correspond to a supersymmetric configuration: neither in the limit of finite compactification radius nor in that in the limit of the chiral momentum.

A detailed calculation of the infrared microscopic degeneracy formula of generic black rings, which correctly 
describes the entropy of MSW black ring solutions, shows in the straight string limit that the expression of the associated entropy of the extremal black rings may be given by

$$
S\left(n_{1}, n_{2}, n_{3}, J_{\psi}\right)=2 \pi \sqrt{n_{1} n_{2} n_{3} J_{\psi}},
$$

which is nothing more than the known leading order large charge macroscopic entropy of the black string. The details of the concerned estimation of the black string entropy may be found in [52]. Hence, we can compute the components of state-space covariant metric tensor defined as the negative Hessian matrix, with respect to the three $M_{5}$-dipole charges $\left\{n_{i}\right\}$ and angular momentum $J_{\psi}$. The associate components of the state-space metric tensor for the entropy of circular black strings may thus simply be given as

$$
\begin{aligned}
g_{n_{1} n_{1}} & =\frac{\pi}{2 n_{1}} \sqrt{\frac{n_{2} n_{3} J_{\psi}}{n_{1}}} \\
g_{n_{1} n_{2}} & =-\frac{\pi}{2} \sqrt{\frac{n_{3} J_{\psi}}{n_{1} n_{2}}} \\
g_{n_{1} n_{3}} & =-\frac{\pi}{2} \sqrt{\frac{n_{2} J_{\psi}}{n_{1} n_{3}}} \\
g_{n_{1} J_{\psi}} & =-\frac{\pi}{2} \sqrt{\frac{n_{2} n_{3}}{n_{1} J_{\psi}}} \\
g_{n_{2} n_{2}} & =\frac{\pi}{2 n_{2}} \sqrt{\frac{n_{1} n_{3} J_{\psi}}{n_{2}}} \\
g_{n_{2} n_{3}} & =-\frac{\pi}{2} \sqrt{\frac{n_{1} J_{\psi}}{n_{2} n_{3}}} \\
g_{n_{2} J_{\psi}} & =-\frac{\pi}{2} \sqrt{\frac{n_{1} n_{3}}{n_{2} J_{\psi}}} \\
g_{n_{3} n_{3}} & =\frac{\pi}{2 n_{3}} \sqrt{\frac{n_{1} n_{2} J_{\psi}}{n_{3}}} \\
g_{n_{3} J_{\psi}} & =-\frac{\pi}{2} \sqrt{\frac{n_{1} n_{2}}{n_{3} J_{\psi}}} \\
g_{J_{\psi} J_{\psi}} & =\frac{\pi}{2 J_{\psi}} \sqrt{\frac{n_{1} n_{2} n_{3}}{J_{\psi}}}
\end{aligned}
$$

One thus observes that the ascertained statistical pair correlations may in turn be accounted for, by simple microscopic descriptions, being expressed in terms of the number (or dipole charges) of the branes and angular momentum connoting an ensemble of microstates of the black string configurations. Furthermore, it is evident that the principle components of the statistical pair correlations are positive definite, for all the allowed values of concerned parameters of the black strings. As a result, we may easily see that the concerned state-space metric constraints are

$$
\begin{aligned}
& g_{n_{1} n_{1}}>0 \forall\left(n_{1}, n_{2}, n_{3}, J_{\psi}\right) \mid n_{1}>0 \\
& g_{n_{2} n_{2}}>0 \forall\left(n_{1}, n_{2}, n_{3}, J_{\psi}\right) \mid n_{2}>0 \\
& g_{n_{3} n_{3}}>0 \forall\left(n_{1}, n_{2}, n_{3}, J_{\psi}\right) \mid n_{3}>0 \\
& g_{J_{\psi} J_{\psi}}>0 \forall \text { admisible }\left(n_{1}, n_{2}, n_{3}, J_{\psi}\right) \mid J_{\psi}>0
\end{aligned}
$$

The principle components of the state-space metric tensor $\left\{g_{n_{i} n_{i}}, g_{J_{\psi} J_{\psi}} \mid i=1,2,3\right\}$ essentially signify a set of definite heat capacities (or the related compressibilities), whose positivity apprises that the black rings comply an underlying local equilibrium statistical configuration. It is intriguing to note that the positivity of the component $g_{J_{\psi} J_{\psi}}$ requires that none of the brane charges and angular momentum of the associated $D_{1}-D_{5}-P$ IR CFT (or the charges in the dual $M$-theory description) should be zero in the IR. This is clearly perceptible because of the fact that the brane configuration becomes unphysical, for these values of the parameters.

It follows, from the above expressions, that the ratios of the principle components of statistical pair correlations vary as the inverse square of the asymptotic charges; while those of the off-diagonal correlations modulate only inversely. Interestingly, we may easily visualize, for the distinct $i, j, k \in\{1,2,3\}$, that the interesting 
statistical pair correlations, thus described, are consisting of the following scaling properties:

$$
\begin{aligned}
\frac{g_{n_{i} n_{i}}}{g_{n_{j} n_{j}}} & =\left(\frac{n_{j}}{n_{i}}\right)^{2} \\
\frac{g_{n_{i} n_{i}}}{g_{J_{\psi} J_{\psi}}} & =\left(\frac{J_{\psi}}{n_{i}}\right)^{2} \\
\frac{g_{n_{i} J_{\psi}}}{g_{n_{j} J_{\psi}}} & =\frac{n_{j}}{n_{i}} \\
\frac{g_{n_{i} n_{i}}}{g_{n_{i} J_{\psi}}} & =-\frac{J_{\psi}}{n_{i}} \\
\frac{g_{n_{i} n_{j}}}{g_{n_{k} J_{\psi}}} & =\frac{n_{k} J_{\psi}}{n_{i} n_{j}} \\
\frac{g_{n_{i} n_{j}}}{g_{n_{i, j} n_{k}}} & =\frac{n_{k}}{n_{j, i}} \\
\frac{g_{n_{i} n_{j}}}{g_{n_{j} J_{\psi}}} & =\frac{J_{\psi}}{n_{i}} \\
\frac{g_{n_{i} n_{j}}}{g_{n_{k} J_{\psi}}} & =\frac{n_{k} J_{\psi}}{n_{i} n_{j}} \\
\frac{g_{n_{i} n_{i}}}{g_{n_{i} n_{j}}} & =-\frac{n_{j}}{n_{i}} \\
\frac{g_{n_{i} n_{i}}}{g_{n_{j} J_{\psi}}} & =-\frac{J_{\psi}}{n_{j}} \\
\frac{g_{n_{i} n_{i}}}{g_{n_{j} n_{k}}} & =-\frac{n_{j} n_{k}}{n_{i}^{2}} \\
\frac{g_{n_{i} n_{i}}}{g_{n_{j} J_{\psi}}} & =\frac{n_{j} J_{\psi}}{n_{i}^{2}} \\
& =1
\end{aligned}
$$

As noticed in the previous configurations, it is not difficult to analyze the local stability for the circular black strings, as well. Radically, one may easily determine the principle minors associated with the state-space metric tensor. Thus, we may demand that all the principle minors must be positive definite. Here, we may adroitly compute the principle minors from the Hessian matrix of the associated entropy concerning the three charge rotating MSW black strings, and thus circular rings. After some simple manipulations we discover that the set of local stability criteria on various possible surfaces and hyper-surfaces of the underlying state-space configuration may respectively be determined by the following set of equations:

$$
\begin{aligned}
& p_{0}=1 \\
& p_{1}=\frac{\pi}{2 n_{1}} \frac{\sqrt{n_{2} n_{3} J_{\psi}}}{n_{1}} \\
& p_{2}=0 \\
& p_{3}=-\frac{\pi^{3}}{2} J_{\psi} \frac{\sqrt{J_{\psi}}}{n_{1} n_{2} n_{3}}
\end{aligned}
$$

For all physically admitted values of the concerned asymptotic charges (or brane number) of black strings/ rings, we may thus easily ascertain that the minor constraint, viz., $p_{2}\left(n_{i}, J_{\psi}\right)=0$ exhibits that the two dimensional state-space configurations are not stable, for any value of the brane numbers and assigned angular momentum. Similarly, the positivity of $p_{1}\left(n_{i}, J_{\psi}\right)$ for arbitrary number of branes shows that the underlying configurations are locally stable, because of the line-wise positive definiteness.

While the constraint $p_{3}\left(n_{i}, J_{\psi}\right)>0$ respectively imposes the condition that the system may never attain stability, for any given positive angular momentum and given positive $n_{i}$ 's. In particular, these constraints enable us to investigate the possible nature of the state-space geometric stability, for leading order MSW black branes. We may thus observe that the presence of planar and hyper-planar instabilities exists for the spinning non-spherical horizon circular black strings. We expect altogether, in the view points of subleading higher derivative contributions in the entropy, that the involved system demands for a restriction on the allowed values of the angular momentum. 
Furthermore, we find that it is easy to enquire about the complete local stability of the full phase-space configuration, which may in fact be acclaimed by computing the determinant of the state-space metric tensor. Nevertheless, it is not difficult to enumerate a compact formula for the determinant of the metric tensor. For the different possible values of brane numbers, viz., $\left\{n_{1}, n_{2}, n_{3}\right\}$ and angular momentum $J_{\psi}$, it may apparently be discovered, from the present intrinsic geometric analysis, that the system finds following expression for the determinant of the metric tensor:

$$
g\left(n_{1}, n_{2}, n_{3}, J_{\psi}\right)=-\pi^{4}
$$

As the determinant of basic state-space metric tensor is a constant and negative quantity, in the large charge limit. Thus, it requires a non-vanishing central charge of the corresponding $D_{1}-D_{5}-P$ CFT in the IR limit, when the associated worldvolume notion of the dual $M$-theory MSW configuration holds [52]. Our analysis herewith discovers that there exists a non-degenerate state-space geometry for the leading extremal MSW configurations. However, it is worth to note that the determinant of the metric tensor does not take a positive definite form, which thus shows that there is no positive definite volume form on the concerned state-space manifold $\left(M_{4}, g\right)$ of the spinning MSW black brane configurations, at the leading order contributions in $M_{5}$-dipoles. This is also intelligible from the fact that the responsible equilibrium entropy tends to its maximum value, while the same culmination may not remain valid on the chosen planes or hyper-planes of the entire state-space manifold. It may in turn be envisaged, in either the $D_{1}-D_{5}-P$ description or dual $M$-theory description, that the black strings/ circular rings do not correspond to an intrinsically stable statistical configuration. Thus, it is very probable that the underlying ensemble of CFT microstates, upon subleading higher derivative corrections, may smoothly move into the more stable brane configurations.

Finally, in order to elucidate the universal nature of statistical interactions and the other properties concerning MSW rotating black branes, one needs to determine definite global geometric invariant quantities on the state-space manifold $\left(M_{4}, g\right)$. Here, we notice that an indicated simplest invariant may be achieved just by computing the state-space scalar curvature, which may indeed be obtained in a straightforward fashion, by applying the formerly explained method of our intrinsic geometry. It turns out that the state-space configuration of the leading order MSW black strings is entirely simple, and in particular, an explicit expression for the scalar curvature is obtained to be

$$
R\left(n_{1}, n_{2}, n_{3}, J_{\psi}\right)=\frac{3}{2 \pi \sqrt{n_{1} n_{2} n_{3} J_{\psi}}},
$$

It is interesting to note that the function $R\left(n_{i}, J_{\psi}\right)$ is nothing more than a regular function, which can completely be defined in terms of the dipole charges and the angular momentum. In this case, the state-space curvature scalar thus remains a non-zero finite function of the dipole charges and angular momentum carried by the circular strings, which physically corresponds to an interacting statistical configuration. We may further observe that the constant entropy curve and that of the state-space scalar curvature take the same form

$$
n_{1} n_{2} n_{3} J_{\psi}=c_{i},
$$

where the real constants $c_{i}:=\left\{c_{S}, c_{R}\right\}$ take obvious values, which may respectively be easily determined for an assigned entropy and state-space scalar curvature. A simple inspection finds, for given entropy $S_{0}$ and state-space scalar curvature $R_{0}$, that the constants $c_{i}$ are defined to be

$$
\begin{aligned}
c_{S} & =\frac{S_{0}^{2}}{4 \pi^{2}} \\
c_{R} & =\frac{9}{4 \pi^{2} R_{0}^{2}}
\end{aligned}
$$

It is worth to mention that the model may also correctly capture the leading corrections, which include the effects of the self-interaction among different points along the ring, that are an effect of the finite ring radius. Thus, the black ring entropy as an expansion of the inverse compactification radius, although not being an independent parameter, may rather be fixed in terms of an explicit combination of the large charges. We thence note that the supersymmetric black rings provide a better behaved statistical system, and in particular, the finite radius effects appear to be absent in underlying state-space configurations of the macroscopic entropy formulae of $[51,52$.

It is thus possible to expose that the black hole non-uniqueness theorems imply a set of important consequences towards an understanding of the state-space description, and thus it is worth to point out the nature of 
Gaussian fluctuations in the MSW ring/ string statistical configurations. In fact, it is a striking, and perhaps a more important deficiency, inherent to the CFT description, that the IR-theory does not allow to say anything about the microscopic significance of black hole non-uniqueness [52, i.e., there are no CFT arguments, how to extract an appropriate set of parameters. Thus, we may find an ambiguity, when dealing with the intrinsic state-space manifold of non-spherical horizon black brane configurations.

Interestingly, we find that the determinant of the metric tensor is non vanishing and thus observe that the underlying state-space geometry describes a non-degenerate intrinsic Riemannian manifold. It may in turn be carried forward to obtain the resulting scalar curvature arising from the degeneracy formula, which correctly describes the maximum correlation volume of the black ring configuration, in the straight string limit. In fact, our analysis easily obtains that the scalar curvature, as a completely regular function of the dipole charges and the angular momentum, turns out to be inversely proportional to the entropy. However, it is expected that, to the leading order in $\alpha^{\prime}$, the invariant quantities would keep a well-defined sign, yielding still a positive definite intrinsic manifold black strings/ ring state-space configuration.

This means, in a sense, that the dipole-based IR theory looks too closely at the ring configuration, and by focusing on the string-like aspects of the ring, it is expected that the state-space configuration is different, with respect to that of the spherical black holes. In order to view both black objects from a unified perspective, we need to further investigate and observe them from greater distances, where one may apply AdS/CFT duality to their concerned ultraviolet CFT descriptions and thus be able to determine an appropriate set of charges/ dipoles and angular momenta, which disport a dominant role in their weakly interacting state-space configurations.

More generally, in order to saturate the BPS bound, supersymmetric black rings necessarily carry conserved $M_{2}$ charges, with integer brane numbers; this, in the microscopic picture, just requires to turn on fluxes on the worldvolume of the constituent branes. These fluxes, though, give rise to zero momentum modes that contribute to the total ring momentum, which balances the momentum available to non-zero-mode oscillators, only if $q_{0}=-J_{\psi}$, see for further details [51,52. This, thus, suggests an intriguing picture for underlying state-space quantities arising from the microscopic entropy obtained from the Cardy formula and objects compared to the Bekenstein-Hawking entropy, and thus yields the underlying nature of inclusive $M_{2}-M_{5}$ limiting configurations.

\subsection{Small Black Rings}

In this subsection, we shall consider the state-space configuration for four parameter small black rings and focus our attention to analyze concerned state-space pair correlation functions in detail. In order to provide an appropriate structure required to accommodate state-space quantities of different black objects with the same conserved charges as those of a class of black rings, it turns out that the supertube, whose state-space configuration shall be analyzed in the next subsection, have the right topology to be identified as the string theory description of limiting small fluctuating circular ring configurations, whose $M_{5}$-contributions we have appraised in the previous subsection.

The statistical analysis of small black rings assumes nothing than the supersymmetric ring, with given two $D$-brane charges $N_{1}, N_{2}$, one dipole charge $n_{3}$, and an angular momentum $J$ for the case of standard $K_{3} \times S^{1}$ compactification and, thence, it turns out that the leading order entropy gives a precise meaning to our statespace considerations. Moreover, Sen et.al. have shown [85] that there exists an underlying microscopic CFT which assigns the same finite microscopic entropy to the small fluctuating circular black ring configurations. Thus, it turns out, in both the microscopic and the macroscopic perspective [52,85], that the entropy of a small black ring respectively depends only on the brane numbers (or related charges) and an angular momentum, viz., $\left\{N_{1}, N_{2}, n_{3}, J\right\}$. It turns out that the entropy, as a function of the connoted parameters, may be expressed as

$$
S\left(N_{1}, N_{2}, n_{3}, J\right):=4 \pi \sqrt{N_{1} N_{2}-n_{3} J}
$$

It is again not difficult to explore the state-space geometry of the equilibrium microstates of the three charge rotating small black ring, arising from the entropy expression which concerns just the Einstein Action. As stated earlier, the Ruppeiner metric on the state-space manifold is given by the negative Hessian matrix of the ring entropy, with respect to the thermodynamic variables. These variables, in this case, are the rotation and conserved brane numbers, which in turn are proportional to the charges carried by the small black ring. Explicitly, we may easily find that the components of the covariant metric tensor are

$$
\begin{aligned}
g_{N_{1} N_{1}} & =\pi N_{2}^{2}\left(N_{1} N_{2}-n_{3} J\right)^{-3 / 2} \\
g_{N_{1} N_{2}} & =-\pi\left(N_{1} N_{2}-2 n_{3} J\right)\left(N_{1} N_{2}-n_{3} J\right)^{-3 / 2} \\
g_{N_{1} n_{3}} & =-\pi N_{2} J\left(N_{1} N_{2}-n_{3} J\right)^{-3 / 2}
\end{aligned}
$$




$$
\begin{aligned}
g_{N_{1} J} & =-\pi N_{2} n_{3}\left(N_{1} N_{2}-n_{3} J\right)^{-3 / 2} \\
g_{N_{2} N_{2}} & =\pi N_{1}^{2}\left(N_{1} N_{2}-n_{3} J\right)^{-3 / 2} \\
g_{N_{2} n_{3}} & =-\pi N_{1} J\left(N_{1} N_{2}-n_{3} J\right)^{-3 / 2} \\
g_{N_{2} J} & =-\pi N_{1} n_{3}\left(N_{1} N_{2}-n_{3} J\right)^{-3 / 2} \\
g_{n_{3} n_{3}} & =\pi J^{2}\left(N_{1} N_{2}-n_{3} J\right)^{-3 / 2} \\
g_{n_{3} J} & =\pi\left(2 N_{1} N_{2}-n_{3} J\right)\left(N_{1} N_{2}-n_{3} J\right)^{-3 / 2} \\
g_{J J} & =\pi n_{3}^{2}\left(N_{1} N_{2}-n_{3} J\right)^{-3 / 2}
\end{aligned}
$$

This framework thus affirms that there exists an intriguing intrinsic geometric enumeration, which describes the possible nature of statistical pair correlations. The concerned state-space pair fluctuations are determined, in terms of the charges and angular momentum of the dipole black rings. Hitherto, the principle components of the statistical pair correlations apparently remain positive definite quantities, for all admissible values of underlying configuration parameters of the black branes. It may easily be observed that the following state-space metric constraints are satisfied:

$$
\begin{aligned}
g_{N_{1} N_{1}} & >0 \forall\left(N_{1}, N_{2}, n_{3}, J\right) \\
g_{N_{2} N_{2}} & >0 \forall\left(N_{1}, N_{2}, n_{3}, J\right) \\
g_{n_{3} n_{3}} & >0 \forall\left(N_{1}, N_{2}, n_{3}, J\right) \\
g_{J J} & >0 \forall\left(N_{1}, N_{2}, n_{3}, J\right)
\end{aligned}
$$

Physically, we may notice that the principle components of the state-space metric tensor $\left\{g_{i i}, g_{J J} \mid i=\right.$ $\left.N_{1}, N_{2}, n_{3}\right\}$ signify a set of heat capacities (or the associated compressibilities), whose positivity exhibits that the underlying black ring system is in a local equilibrium statistical configuration of the branes. Our analysis further complies that the positivity of $g_{J}$ obliges that the associated dual conformal field theory living on the boundary must prevail a non vanishing value of the dipole charge associated with large integers $N_{1}, N_{2}, n_{3}$, which define the degeneracy of the microscopic conformal field theory.

Interestingly, it is worth to note that our geometric expressions, arising from the entropy of small black rings, indicate that none of the brane charges can be safely turned off, say $N_{i}=0$, while having a well-defined state-space geometry. However, it is unfeasible to have an intrinsic black ring state-space configuration with non vanishing charges, say $N_{1}, N_{2} \neq 0$ and $n_{3}=0$, since the objects inside the square-root of the statistical correlations do not take account of the rotation, and thus argued ring configurations with vanishing brane numbers are no more well-defined black rings, but they become spherical horizon small black holes, whose state-state is analyzed in [40].

The ratio of the principle components of statistical pair correlations form two different sets and, specifically, they vary as an inverse square of the two involved parameters; while those of the other off diagonal correlations only vary inversely. It is fundamentally not difficult to inspect, for non-identical $i, j \in\{1,2\}$, that the statistical pair correlations are consisting of the following type of scaling relations:

$$
\begin{aligned}
\frac{g_{N_{i} N_{i}}}{g_{N_{j} N_{j}}} & =\left(\frac{N_{j}}{N_{i}}\right)^{2} \\
\frac{g_{N_{i} N_{i}}}{g_{n_{3} n_{3}}} & =\left(\frac{N_{j}}{J}\right)^{2} \\
\frac{g_{N_{i} N_{i}}}{g_{J J}} & =\left(\frac{N_{j}}{n_{3}}\right)^{2} \\
\frac{g_{n_{3} n_{3}}}{g_{J J}} & =\left(\frac{J}{n_{3}}\right)^{2} \\
\frac{g_{N_{i} J}}{g_{N_{j} J}} & =\frac{N_{j}}{N_{i}} \\
\frac{g_{N_{i} N_{i}}}{g_{N_{i} n_{3}}} & =-\frac{N_{j}}{J} \\
\frac{g_{N_{i} N_{i}}}{g_{N_{i} J}} & =-\frac{N_{j}}{n_{3}} \\
\frac{g_{N_{i} N_{j}}}{g_{N_{i} N_{i}}} & =\frac{1}{N_{j}}\left(2 n_{3} J-N_{1} N_{2}\right)
\end{aligned}
$$




$$
\begin{aligned}
\frac{g_{n_{3} J}}{g_{N_{i} N_{i}}} & =\frac{1}{N_{j}}\left(2 N_{1} N_{2}-n_{3} J\right) \\
\frac{g_{n_{3} J}}{g_{n_{3} n_{3}}} & =\frac{1}{J}\left(2 N_{1} N_{2}-n_{3} J\right)
\end{aligned}
$$

The concerned microstate counting investigations strengthen the fact that the angular momentum $J$ of small black rings is further constrained from below, in term of the brane charges or dipole charges (or numbers), by the bound: $J \leq \frac{N_{1} N_{2}}{n_{3}}$, which defines the reality condition of the entropy [52,85]. Furthermore, it is not hard to find, from the given entropy expression, that the remaining relative state-space correlations retain similar scaling properties. Here, our analysis thus demonstrates that the positivity of state-space pair correlation functions between the non-identical charges $\left\{N_{k}, n_{3} \mid k=1,2\right\}$ stipulates a modified upper and lower bound on the associated angular momentum

$$
\frac{N_{1} N_{2}}{2 n_{3}} \leq J \leq \frac{2 N_{1} N_{2}}{n_{3}}
$$

This is because the brane-brane pair correlations involve the other remaining charges of the underlying dipole ring configuration. Thus, one establishes that the bounds, arising from the stability criteria of state-space pair correlations between the non-identical branes, lie between one half to twice the reality condition of the small black ring entropy.

Note also that our analysis describes a black ring configuration, which is based on the degeneracy of fluctuations around a circular shape of the limiting supertube, with less than the maximal angular momentum, $J<N_{1} N_{2} / n_{3}$. Thus, it corresponds to a definite supersymmetric black ring, which does not suffer from causal pathologies. This condition intriguingly forces us that the angular momentum of the small dipole black string solution should statistically satisfy

$$
\frac{N_{1} N_{2}}{2 n_{3}} \leq J \leq \frac{N_{1} N_{2}}{n_{3}}
$$

Apart from the positivity of principle components of the state-space metric tensor, one further demands that all associated principle minors should be positive definite, in order to accomplish local state-space stability. It is nevertheless not difficult to compute the principle minors of the concerned Hessian matrix of the entropy associated with spinning small black rings. In fact, one encounters, after some simple manipulations, that the local stability conditions on the one dimensional line, two dimensional surfaces and three dimensional hypersurfaces of the concerned state-space manifold may respectively be measured by the following expressions:

$$
\begin{aligned}
& p_{1}=\pi N_{2}^{2}\left(N_{1} N_{2}-n_{3} J\right)^{-3 / 2} \\
& p_{2}=\pi^{2} n_{3} J\left(N_{1} N_{2}-n_{3} J\right)^{-2} \\
& p_{3}=-4 \pi^{3} J^{2}\left(N_{1} N_{2}-n_{3} J\right)^{-5 / 2}
\end{aligned}
$$

For all physically allowed values of large integers defining the brane numbers/ charges of the small black rings, we can stipulate that the minor constraint $p_{2}>0$ obliges that the domain of the ascribed angular momentum must respectively be greater than $J>0$, for a given non-zero $n_{3}$ number of dipoles, while the constraint $p_{3}>0$ never gets satisfied, for any real physical angular momentum and entropy. We may thus proclaim that the angular momentum must be positive real valued, in order to have a definite state-space surface stability condition. As anticipated earlier, we inspect that the nature of the state-space geometry of the spinning small dipole ring configuration is such that there exists planar stability, but its state-space is not stable on three dimensional hyper-planes, for any value of angular momentum.

In addition, it is likewise evident that the local stability of the full small black ring phase-space configuration may be determined by computing the determinant of the concerned state-space metric tensor. Here, we may easily compute a compact formula for the determinant of the metric tensor, and indispensably, our intrinsic geometric analysis provides that the expression for the determinant of the metric tensor, at the leading order entropy analysis [52], does not find an intrinsic state-space value, for any desired brane numbers and angular momentum.

It is, in fact, not difficult to see that the determinant of the metric tensor vanishes identically, for all finite values of the number of branes carried by the small black ring configurations at this order. Thus, the corresponding state-space geometry turns out to be an ill-defined degenerate intrinsic Riemannian manifold. Here, the constant entropy curve is given by

$$
N_{1} N_{2}-n_{3} J=c
$$


where $c=\frac{S_{0}^{2}}{16 \pi^{2}}$ is a known real constant, for a given entropy $S_{0}$. Hence, the state-space geometry based on the large charge equilibrium microstates is trivial, for the leading order small black ring solutions. Also, the present entropy is unworthy, towards an appreciation of the global statistical correlation length. It is worth to mention that the present analysis has been obtained from the brane microstate counting, in the leading nonvanishing entropy approximation, and thus one may anticipate that the subleading orders contributions would possibly facilitate us a definite, non-degenerate intrinsic state-space configuration to the dipole small black rings. Although the associated configuration has a naked singularity instead of a horizon, however it describes the Bose-Einstein condensate of $J$ short strings of length $n_{3}$, in terms of an underlying CFT description of the supertubes, which thus accounts for the angular momentum in a thermal ensemble of strings [86, 87.

The intrinsic geometric understanding of the small black ring and their statistical configurations may further be enhanced by the regularization of the space-time singularity, in the virtues of the underlying string theory/ $M$-theory. Note that our analysis may in turn take account of known specific considerations, and desired higherderivative corrections, as well. It may thus be anticipated that adequate $\alpha^{\prime}$-corrections would ultimately render to well-defined interacting state-space configurations, for the small black rings. This is because the concerned low energy effective action requires nothing more than the framework of standard four dimensional $\mathcal{N}=4$ supergravity techniques.

Most importantly, we notice that the present analysis relies on corrected horizon configurations, when the ring is compactified to four space-time dimensions, by putting it on the Taub-NUT space, which reproduces the familiar Bekenstein-Hawking-Wald entropy of the small black rings, see for instance [87. In turn, De Boer et. al. have shown the existence of a simple model for the dynamical appearance of Bose-Einstein condensates, which describe an intriguing set of creation operators for the short strings of length $n_{3}$ and assigns a dipole of strength $1 / n_{3}$, see 88 for their detailed proposition. Moreover, they have further shown that the onepoint functions of associated dual CFT operators to underlying small black rings are non-trivial. One thus finds that both constructions support an intriguing association between each other. Consequently, the statespace description vindicates physically sound containments of the statistical fluctuations, in small black ring microscopic configurations.

Note further that, if we would like to take an account of the regularization of the singularity in string theory, then it turns out to be plausible by higher-derivative corrections. In the framework of a low energy effective action, it again requires nothing more than the usual techniques of the four-dimensional $\mathcal{N}=4$ supergravity backgrounds. In turn, the modified state-space configurations may be obtained from the corrected horizon, as well, when the ring is compactified to four dimensions by putting it on a Taub-NUT space, which reproduces the Bekenstein-Hawking-Wald configuration of the small black rings [87. Moreover, De Boer et. al. have proposed a simple microscopic model for the dynamical appearance of the Bose-Einstein condensate that describes the creation operators for $J$ short strings of length $n_{3}$, which assigns a dipole of strength $1 / n_{3}$, see the details given in [88. Basically, it may be interesting to see this proposal, how the one-point functions of boundary operators, in the underlying CFT dual of the small black ring, are non-trivial, in our out-set of state-space description.

The state-space configurations of a supertube have, as well, been described by definite values of the specific parameters associated with standard $D_{1} D_{5} P$ CFT. In fact, the present investigation finds that the supertubes are well-defined state-space geometric objects, which we have explicitly exposed for the single bubbled supertube configurations, having identical constituent $D_{1}, D_{5}$ charges, momentum charge and distinct set of dipoles. Thus, in order to consider the framework of $D_{1} D_{5} P$ CFT, one may conveniently start with the black ring configuration and then pass to a different U-duality frame, which yields a set of required co-ordinates on the state-space configuration, and thus the parametric perspective of concerned statistical fluctuations. In precise, the five-dimensional supersymmetric configurations are best understood by, first, uplifting them to the six dimension black strings [28, and then viewing them as an intersection of $D_{1}$ and $D_{5}$-branes, which carry nonzero momentum along their common direction. The state-space correlations associated with the low energies dynamics of the branes may thence easily be described, via the parameters of an underlying $(1+1)$ UV-CFT with central charge $c_{U V}=6 N_{1} N_{2}$, where $N_{1}, N_{2}$ are the number of the $D_{1}$ and $D_{5}$-branes.

Moreover, the underlying statistical configurations of the supertube solutions may further be well-understood, in the framework of sigma-model CFTs, at those points in the moduli manifold, where the target space manifold reduces to a symmetric orbifold of $N_{1} N_{2}$ copies of an internal four-manifold. In turn, one finds that their supergravity description amounts to a deformation of the theory, away from the associated strong coupling regime. Note further, that the UV-CFT contains twisted sectors, as well, at the orbifold point. Accordingly, the statistical configurations associated with the maximally twisted sector physically correspond to a long effective string of length $N_{1} N_{2}$ times the length of the compactification circle that the string wraps. That of the untwisted sector may, similarly, be regarded as containing only the $N_{1} N_{2}$ number of short effective strings. Apart from 
the long and short string configurations, it is worth to notice that there exist also some partially twisted sectors, as well, in the structures of standard $D_{1} D_{5} P$-UV/ IR-CFTs.

One thus finds that the physical properties of underlying state-space pair correlation functions and correlation length acquire an illuminating description for the black strings and black rings; and as a matter of course, this offers how the local and global statistical correlations fit into an appreciation of the parameters of the UV theory. However, there is yet another way of understanding the state-space correlations, in which the angular momentum carried by the $D_{1} D_{5}$ brane configurations can supply one further useful description. One observes wherefore that each individual effective string has a definite fermionic ground state, which can in general be polarized, and thus is allowed to carry an angular momentum $(1 / 2,1 / 2)$ of the $S U(2) \times S U(2)$ rotation group describing the supertube configurations. Furthermore, it is clear, for the ground state configurations in the untwisted sector, that there is a total of $N_{1} N_{2}$ stipulated short strings, which can carry an angular momentum

$$
\begin{aligned}
J_{\psi} & =N_{1} N_{2} \\
J_{\phi} & =0 .
\end{aligned}
$$

One is therefore interested in describing their intrinsic geometric state-space properties. One finds from [18] that the angular momentum is present, even in the absence of momentum excitations. In this case, the concerned ground state as an arbitrary collection of finitely many brane microstates, which correspond to a class of supertubes, whose state-space configurations may be shown to be well-defined and interacting statistical systems over a definite range of the equal $M_{2}$-brane charge.

Note further that the space-time realization of the supertubes is typically accomplished in terms of truly existing tubular configurations of branes, which in the case of single bubbled solutions turn out to be tubes made of single Kaluza-Klein monopole, viz., $n_{3}=1$, and thus possess one lower dimensional state-space manifold. In general, if there are several Kaluza-Klein monopoles with $n_{3}>1$, then the associated state-space geometry, which has presently been considered, deals with the various parameters of tubular configurations of constituent branes and may microscopically be described in specific sectors of the UV-CFT, whose central charge reduces to the value of $c_{U V} / 6 n_{3}$. The state-space configurations restricted in this sector thus deal with those supertubes which involve strings of length $n_{3}$, and an angular momentum

$$
J_{\psi}=\frac{c_{U V}}{6 n_{3}}
$$

For generic supertube configurations, we refer to [15] for further details and the construction of their spacetime geometries. In order to provide the string theory description of the state-space structures required for an accommodation of different black objects with the same conserved charges as that of the supertubes, it turns out that one is obliged to have the right horizon topology, such that the concerned black brane solutions may generically be confronted with the constituents black ring microscopic configurations.

In what follows next, we shall focus on the ring solution that corresponds to taking equal values of the three charges $\left(D_{1}, D_{5}\right.$ and Kaluza-Klein momentum) and three distinct dipoles $\left(D_{1}, D_{5}\right.$ and Kaluza-Klein monopole) of a more general supersymmetric black ring (viewed in higher dimensions, a black supertube [18, or the three charge configurations of [28]). It is worth to anticipate here that the equal-charge solution turns out to be entirely determined by its conserved charge $Q$, but the case does not remain the same for the most general supersymmetric black ring or the black supertubes considered in [17,89, 90. Thus, an understanding of how the microscopic string description of black holes distinguishes between the different horizon topologies has opened an exciting possibility of studying the question of state-space correlations for the regular horizon black holes with finite horizon area. The present case in a supersymmetric, highly controlled setting accounts for the state-space perspective associated with the counting problem and the microscopic existence of the concerned supersymmetric black ring (/string) configurations.

This may further be supported by the fact that the dipole charge may be defined as an integral over the constant coordinates surface outside the $S^{2}$ horizon of black holes. In fact, a given fixed electric charge determines the radius of $S^{2}$ and also the angular momentum of the horizon. It is worth to mention that this charge is not conserved, except in the limit in which the ring becomes an infinite black string. However, the general solutions of [17,89,90] carry three independent dipole charges, which are respectively proportional to the number of $D_{1}$-branes, $D_{5}$-branes and Kaluza-Klein monopoles, with the worldvolume direction around the ring circle $S^{1}$. But the solution of our interest presented here corresponds to taking equal values for the three dipole charges, so the dipole charge is proportional to the number of branes on the worldvolume direction around the ring circle. Moreover, one finds in this case that the black rings, when oxidized to six dimensions, become a class of black supertubes. One may in turn argue that the space-time regularity of this solution leads to the fact 
that the dipole charge is being quantized in units of the Kaluza-Klein compactification radius. An argument follows from [17, i.e. that the dipole charges may be defined by the number of Kaluza-Klein monopoles making up the tube configurations. The next subsection would thus be devoted to the specific state-space configuration involving a unique large brane charge supertubes with three dipoles.

\subsection{Supertubes}

In the present subsection we shall consider state-space manifolds arising from the supertube configurations and analyze the related geometric properties of the underlying statistical pair correlation functions and correlation volume for $M_{2} / M_{5}$-brane configurations. We shall start our analysis with the supersymmetric black rings whose existence is based on illustrious experiments involving three charge supersymmetric black holes and supertubes. Some of the concerned physical motivations arising from the space-time considerations may be found in [28,29. The subsequent discovery shows that there exists an intriguing program to classify five-dimensional supersymmetric black hole solutions of $\mathcal{N}=1$ supergravity. This in turn facilitates us with the supersymmetric black ring solutions, charged black rings and other related configurations with non-uniqueness properties, see for instance [15,75]. It is worth to note that the necessary and sufficient conditions for supersymmetry reduce to a class of simple base space four dimensional manifolds, such that there exists a canonical form for the five dimensional supersymmetric solutions [9,46].

The first supersymmetric black ring solutions have chronologically emerged on the flat base space [89] and their subsequent generalization to $U(1)^{n}$ supergravities are the matter of [17,47, 91. The supersymmetry in fact implies that the mass is fixed by saturation of the BPS inequality and three Killing fields generating black strings with $\mathbf{R} \times U(1) \times U(1)$ isometry group, and thus demonstrates the same configurations as that of the nonsupersymmetric black ring solutions. These solutions depend on seven parameters: dipole charges $d^{i}$, rescaled conserved charges $Q_{i}$ and the length scale $R_{K K}$ corresponding to the radius of the ring, with respect to the base space metric. It is worth to note in the limit $R_{K K} \rightarrow \infty$, with the charge densities $Q_{i} / R_{K K}$ fixed, that the associated ring solutions reduce to black string solutions [29], which are essentially the same configurations as that of the corresponding neutral cases.

The study of bubbled space-time geometries and axi-symmetric merger solutions turns out to be interesting for further investigation, from the view-points of our state-space geometry, whose characterization may be accomplished, in terms of the parameters describing an ensemble of microstates for the supertubes 92 . The authors, Bena et. al. have conjointly shown that the microscopic counting entropy, associated with the single classical black rings, can be expressed as the function of angular momenta, asymptotically measured electric charges and the number of $M_{5}$-branes, which characterizes the microscopic degeneracy of the bubbled black ring configurations. Note further that the classical embedding radius, which is measured in the space-time $R^{2}$-plane, exhibits that the angular momentum $J_{L}$ satisfies the following constraint:

$$
J_{L}=\left(d^{1}+d^{2}+d^{3}\right) R_{K K}^{2}
$$

see [28,29] for the related details of space-time causal pathologies. It may thus be noted that the ring charges and angular momenta of the single bubbled ring solutions can be obtained from possible configurations of the Gibbon-Hawking base points. To simplify the calculation, it is however better to introduce appropriate statespace variables that make the relation between the parameters of bubbling solutions and the supertubes more direct. As anticipated for a single bubbled ring with an identical set of electric charges $Q:=Q_{i}$, one then finds a simple expression for the supertube entropy

$$
S\left(Q, d^{1}, d^{2}, d^{3}\right)=\frac{\pi}{Q} d^{1} d^{2} d^{3} \sqrt{4 Q-1}
$$

We therefore observe, in the classical limit $Q \rightarrow \infty$, that the bubbled ring collapses to the standard ring, and in turn the entropy vanishes corresponding to such a bubbled configuration. The state-space geometry may thus again be defined as the negative Hessian matrix of the supertube entropy, with respect to the asymptotic electric charge and number of $M_{5}$-dipoles. We see in this case that the components of the state-space metric tensor are

$$
\begin{aligned}
g_{Q Q} & =-2 \frac{\pi}{Q^{3}} d^{1} d^{2} d^{3}\left(6 Q^{2}-6 Q+1\right)(4 Q-1)^{-3 / 2} \\
g_{Q d^{1}} & =\frac{\pi}{Q^{2}} d^{2} d^{3}(2 Q-1)(4 Q-1)^{-1 / 2}
\end{aligned}
$$




$$
\begin{aligned}
g_{Q d^{2}} & =\frac{\pi}{Q^{2}} d^{1} d^{3}(2 Q-1)(4 Q-1)^{-1 / 2} \\
g_{Q d^{3}} & =\frac{\pi}{Q^{2}} d^{1} d^{2}(2 Q-1)(4 Q-1)^{-1 / 2} \\
g_{d^{1} d^{1}} & =0 \\
g_{d^{1} d^{2}} & =-\frac{\pi}{Q} d^{3}(4 Q-1)^{1 / 2} \\
g_{d^{1} d^{3}} & =-\frac{\pi}{Q} d^{2}(4 Q-1)^{1 / 2} \\
g_{d^{2} d^{2}} & =0 \\
g_{d^{2} d^{3}} & =-\frac{\pi}{Q} d^{1}(4 Q-1)^{1 / 2} \\
g_{d^{3} d^{3}} & =0
\end{aligned}
$$

Incidentally, we notice from the simple $D$-brane description that there exists an interesting state-space interpretation, which covariantly describes various statistical pair correlation formulae, arising from the corresponding microscopic entropy of the aforementioned supersymmetric (extremal) black brane configurations. Furthermore, our intrinsic state-space pair correlations turn out to be in precise accordance with the underlying macroscopic attractor configurations, being disclosed in the special leading order limit of non-vanishing entropy solutions.

In the entropy representation, we thus see that the Hessian matrix of the entropy illustrates the nature of possible Gaussian correlations between the set of state-space variables, which in this case are nothing more than the brane charges, dipoles, and angular momenta, if space-time causality is violated in general. Substantially, we may articulate for given non-zero values of an identical large electric charge $Q$ and dipole fluxes $\left\{d^{i} \mid i=1,2,3\right\}$, as well, that the $g_{Q Q}$ is only the non-vanishing principle component of the present intrinsic state-space metric tensor. It is thence not difficult to see, for distinct $i, j, k \in\{1,2,3\}$, that the involved component $g_{Q Q}$ satisfies

$$
\begin{aligned}
g_{Q Q}> & 0 \forall\left(Q, d^{i}\right) \mid\left\{Q \in(3 / 2,5 / 2) ;\left\{d^{i}\right\} \text { have alternating sign set }\right\} \bigcup \\
& \left\{Q \in(0,3 / 2) \cup(5 / 2, \infty) ; d^{i} \text { has different sign than } d^{j}, d^{k}\right\}
\end{aligned}
$$

As mentioned before, one finds that the principle components of the state-space metric tensor signifies respective heat capacities, or the associated compressibilities, whose positivity indicates that the underlying statistical systems are in local equilibrium of constituent brane configurations. Furthermore, we perceive that the other allied diagonal components vanish identically, for all electric charge and dipole charges, viz, the dipole-dipole $g_{d^{i} d^{i}}=0$. In contrast, the off-diagonal components do survive in two different sectors. The first one comes as the charge-dipole statistical fluctuation, while the second comes as the distinct dipole-dipole fluctuation. We may, in either sector, see easily that their ratio varies as the inverse of the concerned dipoles $d^{i}$. We further observe that the first set of statistical fluctuations vanish for $Q=1 / 2$, while the second set never vanish, for all finite electric charge $Q$ and non-zero dipole fluxes $d^{i}$.

This configuration thus shows that the brane-brane fluctuations are rather stable over a domain of $Q$, and thus relatively more swiftly come into an equilibrium configuration than those involving the off diagonal ones, which involve brane-dipole or distinct dipole-dipole fluctuations. We may further observe that the ratios of either non-diagonal components vary inversely, and in turn they remain comparable for the longer domain of parameters defining the Gaussian fluctuations, in the entropy of specified single bubbled supertubes. Characteristically, we may easily inspect, in the present case, i.e. $\forall i \neq j \neq k \in\{1,2,3\}$ and for a given $Q$, that the non-vanishing relative off-diagonal statistical pair correlation functions satisfy the following simple scaling relations:

$$
\begin{aligned}
& \frac{g_{Q d^{i}}}{g_{Q d^{j}}}=\frac{d^{j}}{d^{i}} \\
& \frac{g_{d^{i} d^{j}}}{g_{d^{i} d^{k}}}=\frac{d^{k}}{d^{j}} \\
& \frac{g_{Q d^{i}}}{g_{d^{i} d^{j}}}=-\left(\frac{d^{j}}{Q}\right) \frac{2 Q-1}{4 Q-1} \\
& \frac{g_{Q d^{i}}}{g_{d^{j} d^{k}}}=-\left(\frac{d^{j} d^{k}}{Q d^{i}}\right) \frac{2 Q-1}{4 Q-1}
\end{aligned}
$$

Moreover, it should be noted that the behavior of non-identical brane-brane dipole pair correlations $\left\{g_{d^{i} d^{j}} \mid\right.$ $\forall i \neq j\}$ is rather more asymmetric, in comparison with the other possible statistical pair correlation functions. 
This may simply be understood by the fact that the brane-brane interactions impart more energy than either self-interactions or the correlations, for given brane charge $Q$. As a result, we discover that the ratio of branebrane pair correlations, with respect to unique brane-dipole or unique dipole-dipole relative pair correlations, respectively, turn out to be

$$
\begin{aligned}
\frac{g_{Q Q}}{g_{Q d^{i}}} & =-\left(\frac{2 d^{i}}{Q}\right) \frac{6 Q^{2}-6 Q+1}{(2 Q-1)(4 Q-1)} \\
\frac{g_{Q Q}}{g_{d^{i} d^{j}}} & =\left(\frac{2 d^{i} d^{j}}{Q^{2}}\right) \frac{6 Q^{2}-6 Q+1}{(4 Q-1)^{2}}
\end{aligned}
$$

Thus, we deduce that the relative brane-brane correlations vanish exactly at two distinct values of $Q$, which ares not the same as that of the vanishing entropy condition, nor that of the determinant condition. This suggests that the brane-brane statistical correlations remain non-zero and respectively stable under the Gaussian fluctuations, if either of the dipole charge or all of the dipole charges take negative values, or the $Q$ satisfies an inequality

$$
\frac{\sqrt{3}-1}{2 \sqrt{3}}<Q<\frac{\sqrt{3}+1}{2 \sqrt{3}}
$$

or vice-versa with $Q$ not belonging to the above domain. For future applications, it is worth specifying that the end-points of this interval $(a, b)$ have been determined as the roots of the equation $6 Q^{2}-6 Q+1=0$. Similar notions may further be noticed for the relative brane-dipole correlations, i.e. that they remain positive, iff the two transverse dipole charges take the same sign, and they vanish exactly at twice the value of the brane charge, with respect to that of the vanishing entropy condition. Nevertheless, our state-space geometry enjoys lower and upper bounds on the attainable brane charge than the constraint arising from the acquainted large charge supertube solutions. Furthermore, it may, independently of the higher dimensional ring rotations, be inferred from our analysis that the supertube solutions are more strongly saturated than simply the condition of entropy vanishing of the (small) black branes, or the decoupling limit in the AdS/CFT.

An investigation of definite global properties of the general bubbled ring (or supertube) configurations determines a certain stability approximation along each direction, each plane and each hyper-plane, as well as on the entire intrinsic state-space manifold. Specifically, we need to determine whether the underlying supertube configuration is locally stable on state-space planes and hyper-planes, and thus one is required to compute corresponding principle minors of the negative Hessian matrix of the entropy. In this case, we may easily appraise, for all physically likely values of brane charge and dipole fluxes, that the possible principle minors computed from the above state-space metric tensor are given by

$$
\begin{aligned}
& p_{0}=1 \\
& p_{1}=-2 \pi d^{1} d^{2} d^{3}\left(6 Q^{2}-6 Q+1\right)(4 Q-1)^{-3 / 2} Q^{-3} \\
& p_{2}=-\left(\pi d^{2} d^{3}(2 Q-1)\right)^{2}(4 Q-1) Q^{-4} \\
& p_{3}=4 d^{1} d^{2}\left(\pi d^{3}\right)^{3}(Q-1) Q^{-4} \sqrt{(4 Q-1)}
\end{aligned}
$$

Thus, the minor constraints $p_{1}, p_{2}<0$ imply that the supertube configurations under consideration are not stable over the lines, planes of the state-space, for any positive value of the dipole charges. Similarly, the constraint $p_{3}>0$ results in an interpretation that this configuration is stable over three dimensional hyper-planes of the full intrinsic state-space manifold.

Importantly, we may however ascertain the nature of the state-space geometry of supertube systems by saying that the planar and hyper-planar stability exists only in a limited domain of the brane configurations, which arrive whenever one or all of the $d^{i}$ pick up negative real values, or the brane charge $Q$ satisfies $a<Q<b$ and $Q<1 / 4$, respectively, for planar and hyper-planar stability requirement, or vice-versa, such that the minors $p_{1}, p_{2}$ of state-space metric tensor take values over positive reals.

Alternatively, the linear and planar stabilities require that the given supertube configurations are scarcely populated, and thus the net brane charges are effectively bounded by the rescaled conserved charges. Moreover, it is not difficult to investigate the global stability on the full state-space configuration, which may in fact be easily carried out by computing the determinant of the state-space metric tensor. In this case, we observe that the determinant of the intrinsic state-space metric tensor is

$$
g=\frac{\pi^{4}}{Q^{6}}\left(d^{1} d^{2} d^{3}\right)^{2}\left(12 Q^{2}-12 Q+1\right)
$$


which in turn never vanishes, for any given non-zero brane charges, except for the two finite extreme values of the ring electric charge, $Q=\{3 / 2,5 / 2\}$. We may further note that the state-space geometry remains positive definite for almost all allowed values of the electric charge, except in a narrow band when $Q$ satisfies, $3 / 2<Q<5 / 2$. We thus observe that the underlying state-space geometry of bubbled supertubes is in well compliance and, in turn, corresponds to a generic non-degenerate intrinsic Riemannian manifold.

The concerned metric tensor thus defines a well-defined, almost everywhere non-degenerate, positive definite state-space manifold, which may solely be parameterized in terms of the brane charge and dipole fluxes, viz., $\left\{Q, d^{i}\right\}$. We find however worth to emphasize that the determinant of the metric tensor indeed takes a positive definite form, except for a region of a narrow band. Thus, there is no positive definite volume form in this region of the defined state-space $\left(M_{4}, g\right)$. It may thence be concluded that the supertubes, when considered as the bound state of branes in the $D$-brane/ $M$-brane description, do correspond to an intrinsically stable statistical configuration, over a large domain of $Q$.

In order to examine important global properties in these black holes phase-space configurations, one is further required to determine the associated geometric invariants of the underlying state-space manifold. For the present supertubes, the simplest invariant turns out to be the state-space scalar curvature, which may as well be easily computed by using the intrinsic geometric technology defined, as earlier, as the negative Hessian matrix of the entropy captured by the $M_{2}$-brane and $M_{5}$-dipole contributions. Explicitly, we discover that the concerned state-space curvature scalar, for the equal charged single bubbled supertube configurations, may easily be depicted to be

$$
\begin{aligned}
R= & \frac{3 Q}{\pi d^{1} d^{2} d^{3}}\left\{(4 Q-1)^{-1 / 2}\left(12 Q^{2}-12 Q+1\right)^{-2}\right. \\
& \left.\left(120 Q^{4}-240 Q^{3}+126 Q^{2}-18 Q+1\right)\right\}
\end{aligned}
$$

Here, we find that the regular state-space scalar curvature seems to be comprehensively universal for the given number of parameters of the bubbled ring configurations. In fact, the concerned perception turns out to be related with the typical form of the state-space geometry arising from the negative Hessian matrix of the duality invariant expression of the interested black brane entropy.

As a standard interpretation, the state-space scalar curvature describes the nature of underlying statistical interactions of possible microscopic brane configurations, which in particular turn out to be non-zero and well defined, for a large band of the parameters of the chosen supertube solution. Note also that the absence of divergences in the scalar curvature indicates that the present supertube solution is an everywhere statistically stable system as an intrinsic state-space configuration. Thus, it turns out that there are no phase transition(s), or critical lines, or any such commensurable phenomena in the underlying state-space manifold of the black brane supertube solutions, except for the determinant vanishing configurations. Furthermore, we may easily appreciate that the constant entropy curve is a rather standard curve, which may be given by

$$
\left(\pi d^{1} d^{2} d^{3}\right)^{2}(4 Q-1)=c Q^{2},
$$

where $c$ is some real constant for the given value of the entropy of the supertube. This, in fact, determines that the entropy of the bubbled black branes defines a non-degenerate embedding, in the view-points of intrinsic state-space geometry. Moreover, we may also disclose, in the lieu of the present entropy, that the curve of constant scalar curvature is given as

$$
\begin{aligned}
& 9 Q^{2}\left(120 Q^{4}-240 Q^{3}+126 Q^{2}-18 Q+1\right)^{2} \\
& =\pi^{2} k^{2}\left(d^{1} d^{2} d^{3}\right)^{2}(4 Q-1)\left(12 Q^{2}-12 Q+1\right)^{4}
\end{aligned}
$$

Here, the constant $k$ is some real number, which may easily be fixed by giving the value of the state-space scalar curvature. Moreover, it is not difficult to enunciate that the quantization/ duality conditions, existing on the electric and dipole charges, signify a set of general coordinate transformation on the associated state-space manifold, which may be presented in terms of the net respective parameters of the constituent $D$-branes or $M$-branes. We see further that the supertubes or associated ring configurations correspond to a non-interacting statistical system, for the set of electric charges given by the roots of the following quartic equation:

$$
120 Q^{4}-240 Q^{3}+126 Q^{2}-18 Q+1=0
$$

We find, in general, that the state-space geometry $\left(M_{4}, g\right)$ of single bubbled supertube configurations remains well-defined, up to another intrinsic Riemannian manifold, $\tilde{M}_{4}:=M_{4} \backslash\{3 / 2,5 / 2\}$. Thus, there are no critical 
phenomena in the state-space manifold spanned by the supertube parameters, except for the roots of the determinant vanishing condition. This, in turn, indicates that the underlying statistical configuration of the specified supertubes is free from the vacuum instabilities and associated phase transitions.

We find that the state-space geometry of single bubbled supertube configurations defines a well-defined, nondegenerate, curved, intrinsic Riemannian manifold, almost everywhere (except for $3 / 2<Q<5 / 2$ ) in the allowed domain of the parameters. The single supertube state-space configuration thus corresponds to an intrinsically stable, interacting statistical system. It is worth to mention that the bubbled black ring configurations, in the classical limit at which they correspond to the standard classical ring configurations, collapse. Thus, such limits spoil intrinsic geometric properties of the state-space manifold akin to the bubbled ring solutions.

Note further that the corresponding microscopic nature of the state-space manifold may easily be read off, from the position of the GH-points and the flux parameters associated with the shape of the ring blob, under the scaling of the true microstates of the black ring solution, considered in the framework of the abysses and closed quivers. In this framework, it seems interesting to investigate the full bubbled configuration space of the $M$-theory black brane solutions, see for instance [92. Such cases, for example the doubled bubbled black ring configurations, may find an interesting state-space picture, whose entropy has in turn been given by

$$
\begin{aligned}
S\left(Q_{i}, J_{L}, d^{i}\right)= & \pi\left[2 d^{1} d^{2} Q_{1} Q_{2}+2 d^{2} d^{3} Q_{2} Q_{3}+2 d^{3} d^{1} Q_{3} Q_{1}\right. \\
& -\left(d^{1} Q_{1}\right)^{2}-\left(d^{2} Q_{2}\right)^{2}-\left(d^{3} Q_{3}\right)^{2}-3 d^{1} d^{2} d^{3} \\
& \left.\left.-d^{1} d^{2} d^{3}\left\{4 J_{L}+2\left(d^{1} Q_{1}\right)+d^{2} Q_{2}+d^{3} Q_{3}\right)\right\}\right]^{1 / 2}
\end{aligned}
$$

The details regarding the angular momenta $\left\{J_{i}\right\}$, asymptotic $M_{2}$-charges $Q_{i} \in\left\{Q_{1}, Q_{2}, Q_{3}\right\}$ and concerned $M_{5^{-}}$ dipoles $d^{i} \in\left\{d^{1}, d^{2}, d^{3}\right\}$ may be found in 92, which provides a further understanding of the present investigation from different angles.

Nevertheless, as outlined above for a single bubbling supertube configuration, it is easy to check that the state-space geometries apply in more generality, for higher-dimensional black supertubes. The same may as well be accomplished, for a large class of black objects with a more complicated horizon topology. We further expect that these will again match the naive expectation, both in the large $Q_{i}$ limit and in the small $d^{i}$ limit, that the qualitative nature of underlying statistical configurations would presumably have very similar conclusions for the general bubbled backgrounds, as that of the aforementioned singled (or double) supertube solutions.

We may in fact anticipate that a simple state-space singularity resolution mechanism, derived under the present consideration, introduces a set of bounds on the electric charges procured by the supertube configurations. In order to avoid such instabilities in the statistical configurations of bubbled black branes, we must make sure that the state-space manifold, thus associated, must remain well-defined and everywhere regular intrinsic Riemannian manifolds. For the singular state-space manifolds, it is however easy to check that the correlation length diverges and, as we have noted earlier, that in the present case of a single bubbled supertube the state-space scalar curvature as a function of the brane charges, dipole charges and angular momenta, if any, finds a definite change of signature as the function of thermodynamic intrinsic variables.

The underlying expressions of the determinant of the state-space metric tensor and the scalar curvature would also trivially generalize for the case of more complicated $U(1)^{n}$ or $S U(n)$ supergravity theories. The general properties of the state-space geometric equations thus obtained shall presumably be quite involved, however they have some rather interesting qualitative features. The detailed explications of these points are however left out for future exploration. While we have not been able to analyze a general supertube configuration in a true sense, we however suspect that the positivity of the determinant of the state-space metric functions would directly follow from the bubble equations, triangle inequalities, and some other simple constraints on the brane and dipole charges of the considered supertube solutions.

Moreover, the supertube state-space configurations indicate that the underlying thermodynamic state-space geometry arising form the Hessian of the corresponding entropy entails an interesting statistical interpretation, i.e. that they are just state-space pair correlation functions, which support a definite definition of the concerned heat capacities (or associated compressibilities). In most of the cases, the statistical configurations of supertubes can be understood from our geometric expression involving a large number of $M_{5}$-brane dipoles and $M_{2}$-brane charges, which in general make up a rotating supertube, with an angular momentum of the tube alone. It is worth mentioning further, in the case of zero-entropy black rings, that the concerned angular momentum may completely be determined in terms of the compactification radius. In fact, as we have advertised in the previous subsections, our conclusions thus match exactly with the bubbling supertube radius and angular momenta, both in the limit when the dipole charges are small, and in the limit of large electric (or $M_{2}$-brane) charges.

The coordinate transformations on the state-space manifold being defined in terms of the brane parameters imply that one supergravity solution exists, with two different brane interpretations. Furthermore, the same 
is present in all the other ring systems, as well, whichever contains certain branes wrapped on topologically trivial cycles, and thus seems to offer definite key findings of the state-space geometry, which characterizes the statistical nature of fluctuating bubbling rings and supertubes. Hence, our state-space investigations, up to the coordinate transformations, show that the bubbling solutions are identical to the naive solutions, which physically happens at distances much larger then the size of the bubble. Moreover, in the classical limit when the $M_{2}$-brane charges $Q \rightarrow \infty$, or in the limit of small number of $M_{5}$-branes, one finds that the investigations thus realized, are further supported by the fact that the bubble configuration, which is nucleated to resolve the existing space-time singularity of the three charge supertubes, become very small, and the resolved solution becomes virtually indistinguishable from the naive brane solution [93. This confirms the intuition coming from the discussion of geometric transitions, BPS geometries and similar other fundamental phenomena.

Finding the supergravity solutions of the three-charge supertubes of arbitrary shape is quite involved. On other hand, it has however been shown in [4] that one can solve underlying equations of motion for these configurations in a linear fashion [9,46, 47, and may further reduce the whole problem of finding three-charge BPS solutions to the standard electromagnetism in four dimensions. In fact, there exists a side-effect of the study of three-charge supertubes [28, 29] and the subsequent discovery [17, 47, 89, 91] of BPS black rings, which by themselves have opened up a new window into the prediction of the black-hole physics $4,48,49,51,77,81,84$, 90, 94 97. A future analysis is thus needed to find the nature of statistical fluctuations beyond the Gaussian approximation, in order to find general stability criterions in generic bubbled black brane configurations. This would also indicate an interesting feature of predisposed statistical fluctuations, i.e. whether an equilibrium black brane solution truly remains stable and does not suffer from (vacuum) instabilities.

\section{Conclusion and Discussion}

The present study considers the intrinsic state-space geometry arising from the fluctuating non-spherical horizon higher dimensional rotating black brane solutions, and we have exemplified our out-set for the string theory and $M$-theory configurations. It is instructive to note that our state-space investigations are based on an understanding of the microscopic entropy of diverse black brane configurations, in which the present consideration requires the coarse graining phenomenon of a large number of degenerate CFT microstates defining an equilibrium statistical system. An appropriate analysis thus finds that the crucial ingredient in analyzing the state-space manifold of rotating black brane configurations depends on the parameters provided by an underlying microscopic conformal field theory. Our illustration of the state-space geometry of higher dimensional black branes includes the case of $D_{1} D_{5} P$ black branes, circular black rings, small black rings, and supertube configurations. In such cases of the non-spherical horizon black strings and black rings, we have thereby focused our attention on the stability constraints arising from the analysis of state-space pair correlation functions and global correlation volume.

The state-space geometry thus described may further be shown to exhibit the associated properties and in particular, one may analyze the possible physical nature of the state-space geometric correlation functions and the correlation volume of concerned statistical configurations. It is worth to mention that the components of the state-space metric tensor are related to the two point statistical correlation functions, which are in general intertwined with the fluctuating parameters of the associated boundary conformal field theory. This is because the required parameters of black brane configurations, which describe the microstates of dual conformal field theory living on the boundary, may in principle be determined via an application of the AdS/CFT correspondence. In this way, our intrinsic geometric formalism, thus described, deals with an ensemble of degenerate CFT ground states, which at an amusingly small constant positive temperature form an equilibrium vacuum configuration, over which we have defined the Gaussian statistical fluctuations.

It is interesting to note that the quadratic nature of Gaussian statistical fluctuations, about an equilibrium statistical configuration, determines the metric tensor of associated state-space manifolds. In either cases, an explicit computation shows, over the domain of black brane parameters, that the principle components of statespace metric tensors are positive, while the non-identical off-diagonal ones may not be so. Furthermore, in order to appreciate definite global properties of the concerned configurations, one is required to determine the nature of stabilities along each direction, each plane, and each hyper-plane, as well as on the entire intrinsic state-space configuration.

Our analysis has demonstrated that the determinant of the metric tensor may as well be negative definite, for the exemplified higher dimensional black strings and black ring configurations. It is however in perfect accordance with the known fact of the Ruppenier geometry that only the classical fluctuations having a definite thermal origin deal with the the probability distribution, which has a positive definite invariant intrinsic 
Riemannian metric tensor, over an equilibrium statistical configuration. In fact, our state-space construction, for the non-spherical horizon black holes dealing with the parameters of microscopic CFTs, clearly illustrates that the degeneracy and the signature of the state-space manifold can be indefinite and sensitive to the location chosen in the moduli space geometry.

Moreover, the absence of divergences in the scalar curvatures imply that the considered black string/ ring solutions are thermodynamically stable, and vacuum phase transitions may be characterized in these configurations. The present investigation thus serves as a prelude to the state-space geometry of arbitrary non-spherical higher dimensional black brane configurations. Intimately, we have explicated that the examples thus explored have an interesting set up of intrinsic state-space geometry, which describes the nature of quadratic fluctuations associated with the statistical configuration of rotating black branes. A straightforward contemplation finds in general that it is trustworthy to categorize these configurations in the following cases.

1. the underlying configuration turns out to be everywhere well-defined, whenever there exists a non-zero state-space determinant.

2. the underlying configuration corresponds to an interacting statistical system, whenever there exists a nonzero state-space scalar curvature.

The main line of thought which has been followed here was, firstly, to develop an intrinsic Riemannian geometric state-space geometry conception to underlying leading order statistical interactions, existing among various CFT microstates of rotating black brane configurations in string theory and $M$-theory. The promising perspective of concerned notions thus arise from the negative Hessian matrix of the corresponding coarse graining entropy, defined over an ensemble of a large number of brane microstates, characterizing the considered rotating non-spherical black hole configurations. Importantly, we have investigated whether the associated state-space geometries are non-degenerate and imply an interacting statistical basis for these configurations, like the one above for instance. The state-space configuration of supertubes has intriguingly been described by well separated Gibbon-Hawking charges, with vanishing total Gibbon-Hawking charge. We further divulged that the behavior of statistical pair correlations, among the equilibrium microstates, may be governed by the consistent parameters defining concerned microscopic CFT vacuum configurations. Thence, the same remains valid for the other associated intrinsic geometric quantities, as well, on the respective underlying state-space manifolds.

In this article, we have explained how particular statistical pair correlation functions, associated with an ensemble of CFT microstates, characterize the behavior of state-space manifolds of the higher dimensional rotating black string and black ring configurations. Interestingly, it has been admissible to investigate whether the state-space correlations, as the function of desired brane numbers or charges on them, are positive definite and regular/ singular function on the respective state-space manifolds. Moreover, in the case of a singular state-space scalar curvature, we have analyzed the nature of concerned singularities, and thus presented them as critical curves on the underlying state-space equilibrium manifolds. Thereupon, we have shown that there exists definite critical points, critical lines, and critical (hyper)-surfaces, which may all be defined by the divergence structure of underlying state-space scalar curvatures.

After having a detailed discussion of non-spherical horizon topology black holes, we have explicated the nature of their underlying state-space configurations. In this concern, the preliminaries which have been motivated in [3] have been extended for an explication of generic state-space pair correlation functions, in general. For example, the $D_{1}-D_{5}-P$ black strings and small black strings indicate degenerate state-space geometry, while the circular strings and supertubes have non-definite state-space configuration. It is nonetheless expected that an appropriate contribution of higher derivative corrections would facilitate numerous black strings/ ring configurations, having well-defined positive definite state-space geometry. The higher order $\alpha^{\prime}$-corrections, when taken into account in the underlying effective theory, are envisaged to offer diverse well-defined state-space configurations or, at least, the non-degeneracy of state-space configurations may generically be expected, in comparison with the ill-defined state-spaces obtained for the $D_{1}-D_{5}-P$ black strings and small black strings, at the leading order entropy solutions. In addition to this, similar conclusions have been established for the heterotic small black holes [40, and thus we contemplate that the black strins/ rings should acquire a well-defined, non degenerate and curved state-space geometry. There are however many caveats, many things which require further clarification and many open questions, and we leave such issues for the future.

The present article analyzes the case of general higher dimensional configurations and thereby shows that the black strings/ rings may not have well-defined state-space manifold, in general. Some of the arguments are briefly based on the nature of parameters, which characterize a countably large number of degenerate equilibrium microstates and thus define concerned rotating black brane configurations. The specializations made are analogous to those mimicked in 41, 42. Here, we have defined the notion of statistical interactions 
in the context of bubbling AdS geometry and other well-known black string and black ring configurations. In section 2, we have specifically developed what are the relative state-space pair correlation functions for given configuration. We have explicitly constructed general features of our state-space geometric covariant quantities, which come from an underlying black brane entropy, and then discussed them with those that arise from their naive microscopic investigations.

The state-space manifold thus described primarily out-lines the stability criteria over the range of parameters defining the concerned higher dimensional black brane configuration, with or without rotations. Indeed, we find that the intrinsic geometric implications arising from the state-space construction are stunning. In particular, they provide a unified framework to analyze both the statistical correlations and singularities, which in general may exist in arbitrary finitely many parameter black brane configurations. In this case, we notice definite interesting supergravity configurations, satisfying well-definiteness condition for the state-space geometry, and thus supplying a globally well-defined positive definite volume form to the concerned state-space manifold. It has explicitly been shown in the large charge limit, where underlying computations of the entropy are valid, that the supertube configurations have a positive definite metric tensor and a specific scalar curvature. Our exposition thus provides simple enough techniques to disclose the nature of fluctuating black string and black ring configurations.

Note that state-space conclusions, in general, depend on the attractor fixed points of the underlying moduli space configuration, and thus they can vary over basin to basin, or away from either attractor fixed point(s) of chosen black brane attractor solutions. The present analysis nevertheless finds ground in the leading order contributions, and it turns out that the obtained certitudes remain valid under the perturbative approximation of the black brane entropy, in a chosen attractor basin. It may further be expected that nothing special would happen, and the number of degenerate CFT states forming the vacuum does not change, over the domain of the parameters of large black brane configurations. Another important ingredient in the present discussion of statespace geometry has been the fact that there exists a large class of rotating extremal black branes, non-extremal black branes, multi-centered black branes and various other tenable black brane configurations in string theory and $M$-theory, which offer several interesting physical implications. It may also be envisaged that the statespace geometry may interestingly find parametric implications with other developments, and the AdS/CFT correspondence seems perfect, in this regard, that it may offer an adequate account of the fluctuations among an ensemble of brane microstates of rotating black branes and thus their associated state-space configurations.

\section{Black String and Black Ring Intrinsic State-spaces}

The state-space geometry of five dimensional supersymmetric black ring solutions may in general be described by five independent conserved charges, namely, the $D_{1}, D_{5}$ brane charges and momentum charge (determining the mass through the saturation of the BPS bound), and two independent angular momenta. In an akin limit of charges, one can easily make contact with the string theory or $M$-theory, by arguing that the net charges and dipole charges must be large quantified integers, since they represent the number of branes and units of Kaluza-

Klein momenta. As the consequence of charge quantization, we thus observe that the finite violation of black hole uniqueness theorems, for non-spherical horizon solutions, would in fact simply enter in their state-space geometric properties.

Moreover, it may be observed that the present study relies on the analysis of the entropy of black strings and black rings, which in the original string theory computations 98 may easily be accounted for those microstates, which have the same conserved charges as that of the concerned black brane configurations. However, the entropy calculation shows that the black ring configurations always have an unequal amount of angular momenta, and thus the rings themselves go on the right track with the proposal of [15], and thus with their state-space geometric investigations, what we have been analyzing here and exploring for the future. The concerned features may essentially be elucidated from the fact that we should focus on the counting of only those microstates, which belong to the specific sectors of $D_{1}-D_{5}$ CFT, and in turn, the precise sector may be determined by assigning definite values to the dipole charge(s) and angular momenta.

On other hand, we find that the black brane configurations describing black rings and black strings indicate similar statistical pair correlations and intrinsic geometric properties to those of the spherical horizon black holes [3. In fact, the present analysis is surmised to provide positive evidence for physically significant identifications associated with the attractor mechanism, and that of the microstates counting of the chosen configurations. In short, the present study provides an introduction to the state-space of black branes in general relativity, string theory and $M$-theory. The very purpose of present the inquiry, thus, explicates the typical nature of state-state configurations, which arrive with the five-dimensional black holes, of $S^{1} \times S^{2}$ event horizon topology. Novel 
aspects of our presentation further include an intriguing approach to construct state-space manifolds, arising from the parameteric fluctuations of black ring/ black strings, i.e. that an ensemble of CFT microstates of chosen black branes furnish a critical reconnaissance of the underlying equilibrium state-space configuration.

An existence of supersymmetric black ring configurations thus raises a set of interesting questions, for an investigation of the intrinsic state-space geometry. For example, the present needs may worthfully be examined, by considering

1. what happens to the state-space manifolds, for the supersymmetric black holes of the minimal supergravity solutions in the empty regions of the $J_{\phi^{-}} J_{\psi}$ plane, or any other solutions which overlap with the covered regions?

2. what are the implications of state-space manifolds, with the full uniqueness theorem being strengthened by the [99, for the supersymmetric black holes and black supertubes?

It would also be important to see whether this kind of approach can be pushed further to general non-extremal black ring solutions, which reproduce the above solutions as a special case; and those of the [13, 14, which presumably depend on the five parameters corresponding to angular momenta, electric-magnetic charges, dipole charges, in general, and mass of the black string and black ring configurations under the question. Finally: is it possible to perform detailed statistical mechanical or CFT calculations, against the state-space pair correlation functions and correlation volumes associated with the extremal black string and black ring solutions? It would further be instructive to extend our analysis for the most general non-extremal and/ or non-supersymmetric black brane configurations. In order to appreciate the present consideration, what we have focused in this paper may in general be analyzed for generic black strings, black rings and supertubes. In the next section, we shall thus offer a set of prospective issues, for the state-space configurations of general $D_{1}-D_{5}-P$ and $M_{2}-M_{5}$ configurations.

\section{$6 \quad$ Future Directions and Open Issues}

Promising explorations of state-space geometry to finite parameter black string/ ring configurations in string theory and $M$-theory offer the definite explicit nature of generic non-spherical horizon black strings and black rings. Before concluding this article, we thus wish to address some recent developments in the string theory and $M$-theory. The rest of this section would focus its attention on very general interests, possible implications and analyze some of them, from the perspective of our intrinsic state-space geometry.

\section{(i) State-space Instabilities and dual CFTs}

Definite progresses have been made towards the construction of 3-charge solutions describing black ring microstates, and in particular the method of adding a small amount of Kaluza-Klein momentum, as a perturbation to 2-charge supertubes results in the 3-charge solutions of 100. In this paper, we have investigated underlying parametric local and global statistical correlations, for the generic black string/ ring configurations under specific considerations, and thereby constructed their related state-space geometries. Furthermore, our state-space description is expected to provide the nature of a large class of three charge ring solutions, which are non-spherical horizon black holes, whose microstates have been proposed and studied in the footings of [27,76, 78, 101. In fact, such an analysis accounts for the covariant description of the configurations being considered in Refs. [76, 101. Upon applying aforementioned techniques, one can explicate the nature of multicenter Gibbons-Hawking solutions, whose base space manifold deals with various poles of positive and negative residues.

These solutions describe rich topological structures related to the dipole sources resolved into fluxes, along new internal cycles of the moduli configurations. However, the mapping between these supergravity solutions and their dual CFT states has not really been properly identified in pertaining cases in the existing literature. Thus, several akin micoscopic meanings of the state-space geometric investigations are yet stimulated to be examined into the particulars of the associated dual CFTs and of the microscopic duality symmetries. The issues like string duality symmetries and their macroscopics interplay with our parametric intrinsic state-space geometry have been left out for the associated general rotating black brane configurations appearing under the string theory and $M$-theory considerations. As for the limiting configurations being divulged in this paper, we may nevertheless ratify that the pertinent instabilities, if any, would precisely occur at the divergence points, curves, and hyper-surfaces of their state-space geometric invariants of the generic rotating and non-rotating black brane solutions. 
Qualifications of dual CFTs and microscopic symmetries deserve further study and, in turn, they leave many open possibilities, under which the black brane configurations can acquire various instabilities in the domain of their parameters. It is worth to mention, thereupon, that the dipole charges of given black black brane configurations may help to stabilize them against some local and/ or global perturbations, like GL modes, chemical potential fluctuations corresponding to the electric-magnetic charges and dipole charges, rotational fluctuations corresponding to the angular momenta, and the thermodynamic temperature fluctuations for the near-extemal and non-extemal black brane solutions. Thence, a large class of such affiliated instabilities, connoted by general rotating black brane configurations in higher space-time dimensions, deserve further study.

\section{(ii) D Dimensional Black Brane Configurations}

We further see that number of parameters which characterizes a black brane configuration increases with the space-time dimension $D$, and thus the degrees of freedom of gravity increase accordingly. It is therefore natural to expect more complex dynamics possessing more parameters of the solution, and thus suggesting a relatively higher dimensional state-space manifold, when the space-time dimension $D$ grows. It is known that the standard $D=4$ gravity is highly constrained, due to the black hole uniqueness theorems, and thus one finds that the only rightful parameters are the mass, charges, and angular momentum, which completely parameterize the existing black hole space-time solutions. It is thus anticipated that one may easily define and compute concerned invariants of underlying state-space manifolds and in principle analyze the possible statistical nature of the known $D=4$ black holes $3,38,40,42$.

The discovery of black strings/ rings shows however that $D=5$ solutions allow more freedom. Their spacetime dynamics is still amenable to some additional dipole charges, in contrast to that of the spherical horizon black hole solutions of $D=4$ gravity. In this paper, we have performed a detailed study of the underlying statespace manifolds, for various contrivable possible cases of their parameters, which characterize chosen black brane configurations and arise as the consistent lower dimensional solutions, in the view-points of string theory and $M$-theory compactification(s). It is thus natural to wonder what would be the story for the $D>5$ gravity configurations, which largely remain unexplored, although there have been some indications that black holes (even spherical horizon ones) may possess qualitatively unexpected features [3, 102]. The concerned issues for the general black brane configurations would thus be the matter of future investigations.

It would indeed be interesting to analyze concerned behaviors of the associated state-space manifolds, for various black rings of horizon topology $S^{1} \times S^{D-3}$, for $D>5$. Moreover, we would like to explore what happens to them, with some other horizon topologies in $D>5$. For example, the consistent higher dimensional horizon topologies, which turn out to be the cases of $S^{1} \times S^{1} \times S^{2}, S^{3} \times S^{3}$, are the open possibilities, in accordance to the higher-dimensional horizon topology theorem [103. Physically speaking, the dimension-dependent gravitational force decays faster with the distance, so it would play a dominant role only around an equilibrium statistical system for the small radii rings. In turn, it has been suggested that the thin black rings should thus exist in $D>5$, as in standard $D=5$ solutions, and they should also be unstable against radial perturbations [103, 104. We hope here that these notions may incidentally be explained from the divergence structure of underlying state-space geometries.

Observe further the fact that there is no bound on the angular momentum, even for the Myers Perry black holes with single spin in $D>5$ [104, and so an existence of higher dimensional rotating black strings/ rings would automatically imply a definite violation of the black hole uniqueness properties. This in turn implies that the underling higher dimensional state-space manifolds would relatively be parameterized by a large number of dipole charges, which live on an equal footing with the other globally conserved parameters of candid holes, rings, tubes,... and other pertinent configurations. Thus, there indeed exists the definite possibility of an even larger class of instabilities in the higher dimensional black brane configurations, which need to be understood in the framework of our state-space geometry.

\section{(iii) Bubbling Black Brane Solutions}

The physics of space-time singularity resolution is very preeminent to the present state-space motivation. In fact, what has been inspired by the investigations of Lin, Lunin and Maldacena (LLM) 93] finds a sound physical consideration in which the bubbling solutions reduce to naive giant gravitons in the small dipole charge limit, and thus have topological transitions where constituent branes are replaced by dissolved fluxes. It is important to note that the indicated black branes, having well separated Gibbon-Hawking charges and vanishing total Gibbon-Hawking charge, have non-degenerate and curved state-space configurations, whose intrinsic metric 
tensor, as a result, determines heat capacities. At the same time, the associated scalar curvature describes the global nature of underlying statistical systems. Thus, it should not be difficult to establish the behavior of state-space correlations, about an equilibrium statistical configuration, as an ensemble of CFT microstates. Intriguingly, we envisage that the set of parameters would form a co-ordinate chart for the concerned intrinsic state-space manifold.

One finds, in this spirit, that the state-space geometries associated with the bubbling black brane solutions become rather hard to analyze, when the number of distinct dipole charges becomes large enough. This observation follows further from the well-known LLM construction [93, i.e. that in this limit the underlying configurations have no obvious brane interpretations, and thus there may not be an illusive set of parameters which define their state-space configuration. More generally, the state-space outcomes are potentially interesting, for analyzing relevant structures of the vacuum manifold associated with the supersymmetric vacuum states in string theory. This seems to be based on the fact that proving or disproving the strong form of Mathur's conjecture [73, which states that black hole microstates are dual to smooth supergravity solutions, finds stunning consequences and gives rise to our state-space geometries, in the limit of Gaussian approximations.

It known that the Mathur's conjecture reduces to a definite well-defined mathematical problem of classifying and counting asymptotically flat four dimensional hyper Kähler manifolds, which have moduli regions of signature $(+,+,+,+)$ and $(-,-,-,-)$, see for instance [76. Note further, from the existence of a weak form of the Mathur conjecture 22, 73, which states that the black hole microstates are dual to string theory configurations with unitary scattering, that are not necessarily smooth in the supergravity approximation, may provide a better understanding of the state-space geometric objects, and thus the statistical fluctuations in them. Thus, the Mathur's conjecture, if correct, indicates that the black holes can be contemplated as an ensemble of hyperKähler geometries, involving foams of a very large number of topologically non-trivial 2-spheres, threaded by fluxes. Hence, our state-space geometric investigation seems to find several microscopic interpretations. Interesting state-space inter-relations and their Kähler geometric or moduli space details are nonetheless beyond the scope of the present set-up, and thus are the matter of future explorations.

\section{(iv) Generalized Hyper-Kähler Manifolds}

It is worth stressing that the possibility of negative determinants of state-space geometries is not really a serious problem, and in fact the positive definite condition is, as such, not mandatory. This is because the signature of the base-space manifold is allowed to change in the presence of non-zero dipole fluxes, so that it should give rise to smooth three charge space-time geometries. This fact may further be explained on the basis of the classification of generalized hyper-Kähler manifolds, as well. Nevertheless, we do not find it necessary to explicate it here, for the purpose of state-space geometry, and for developing some interesting physics of black holes/ black strings/ black rings.

Furthermore, the black hole microstates may be described by a set of foams of non-trivial $S^{2}$ 's over the four-dimensional base manifold [76, so one may be able to carry out some statistical analysis, as well, for such foams, perhaps by using a toric geometry, to see if one can describe macroscopic, bulk state-space pair correlation functions and correlation volume of these black brane configurations. It would also be interesting to investigate the contributions coming from the transitions between different base-space geometries, nucleation of GH points, probabilities of transition, and the instanton corrections, into our state-space manifolds. It is worth to note at this point that the idea associated with the state-space manifolds appears to be arising from the space-time foams, which may be important in understanding divergence structures of the state-space/ base-space/ moduli space manifolds, as the space-time foam has made regular appearances in the discussion of quantum gravity, see for example [105.

In a very general sense, what we are interrogating here is, in somewhat similar spirit to the notions which deal with the correlation functions in QFT or the fluctuation theory in statistical mechanics, that they can be divulged from the fact that the underlying brane space-times on small scales become inevitable fuzzy topological foams [76. Here, we have however managed to find state-space correlations, in the limit of supersymmetric D-brane configurations, and with these outcomes to a great deal, more computational control of the problem is required, to be physically understood. Categorically, one needs to find how the equilibrium microstates interact each other, under the Gaussian fluctuations. These points, and their non-equilibrium generalizations, are beyond the scope of the present interest, and thus we leave them to open up in other considerations. 


\section{(v) Physics at the Planck Scale}

It is important to note that the same physical ideas, which led to an apprehension that the space-time becomes foamy near the Planck scale, also come into an interplay in the state-space considerations. This is because, at the Planck scale, there are virtual black holes even in empty space, and we are thus analyzing the underlying statistical correlations among the microstates, which may be described by the aforementioned foam of twospheres. The concerned connotation of thermodynamic state-space geometry may be indicated from [71, 72]. Consistency requirements would therefore suggest that the local and global state-space correlations of existing virtual black holes should really be virtual fluctuations in bubbling hyper-Kähler geometries. Therefore, even empty space would have definite state-space interactions, which we have been exploring here Thus, they should apparently have some generalization, in terms of the parameters of foamy geometries.

Obviously, the state-space geometric description will naturally break down at the Planck scale, but the picture is expected to remain rather interesting, and still it is certainly supported by the fact that large bubbles [28, 29, 93] are needed to resolve singularities persisting in the macroscopic black brane configurations. There are evidently many things to be tested and lots of interesting things might be done, but we believe that we have made definite important progress, by divulging the parametric state-space manifolds and, thereby, have given an intriguing description to local and global state-space correlations existing among black hole equilibrium microstates. The present exploration thus opens an avenue and may give new insight into the promising structures of black brane space-time on very small scales.

\section{Acknowledgement:}

The work of S. B. has been supported in part by the European Research Council grant n. 226455, "SUPERSYMMETRY, QUANTUM GRAVITY AND GAUGE FIELDS (SUPERFIELDS)". B.N.T. would like to thank Prof. R. Emparan, Prof. A. Sen and Prof. J. de Boer for useful discussion on the stability of rotating brane configurations and phase transitions during the "Spring School on Superstring Theory and Related Topics-2008, ICTP Trieste,Italy"; Prof. J. Simon for exciting discussions on BPS configurations and their microscopic models during the "School on Attractor Mechanism, June-July 2009 (SAM2009), INFN, Frascati, Italy"; Prof. V. Ravishankar for providing viable support during the preparation of this manuscript; Dr. B. Bhattacharjya and Dr. V. Chandra for a set of discussions on the phases of intrinsic Riemannian and thermodynamic geometries, while this manuscript was under preparation. B.N.T. also acknowledges "CSIR, New Delhi, India", for the financial support under the research grant "CSIR-SRF-9/92(343)/2004-EMR-I" and the international travel supports of the "Indian Institute of Technology-Kanpur, Kanpur-208016, India" towards the participation in the "STRINGS-2009, Roma" and "SAM2009 at Frascati, Italy", where the part of this work was done.

\section{References}

[1] S. Bellucci, B. N. Tiwari, "An exact fluctuating 1/2-BPS configuration," JHEP 05 (2010) 023, arXiv:0910.5314v2 [hep-th].

[2] S. Bellucci, B. N. Tiwari, State-space correlations and stabilities, Phys. Rev. D 82, 084008, (2010), arXiv:0910.5309v1 [hep-th].

[3] S. Bellucci, B. N. Tiwari, "State-space Manifold and Rotating Black Holes", [Communicated], arXiv:1010.1427v1 [hep-th].

[4] S. Ferrara, K. Hayakawa, A. Marrani, "Erice Lectures on Black Holes and Attractors", Fortsch. Phys. 56, 993-1046, 2008, arXiv:0805.2498v2 [hep-th].

[5] B. L. Cerchiai, S. Ferrara, A. Marrani, B. Zumino, "Duality, Entropy and ADM Mass in Supergravity", arXiv:0902.3973v2 [hep-th].

[6] S. Bellucci, S. Ferrara, A. Marrani, A. Yeranyan, "stu Black Holes Unveiled", Entropy 2008 Vol. 10 (4), p. 507-555, arXiv:0807.3503v3 [hep-th].

[7] S. Bellucci, S. Ferrara, A. Marrani, "Attractors in Black", Fortsch. Phys. 56, 761-785,2008 arXiv:0805.1310 1 [hep-th].

[8] J. B. Gutowski, H. S. Reall, "Supersymmetric $\mathrm{AdS}_{5}$ black holes", JHEP 0402, 006 (2004), arXiv:hep-th/0401042 
[9] J. B. Gutowski, H. S. Reall, "General supersymmetric $\mathrm{AdS}_{5}$ black holes", JHEP 0404, 048 (2004), arXiv:hep-th/0401129

[10] Z. W. Chong, M. Cvetic, H. Lu, C. N. Pope, "Five-dimensional gauged supergravity black holes with independent rotation parameters", Phys. Rev. D 72, 041901 (2005), arXiv:hep-th/0505112

[11] Z. W. Chong, M. Cvetic, H. Lu, C. N. Pope, "General non-extremal rotating black holes in minimal five-dimensional gauged supergravity", Phys. Rev. Lett. 95, 161301 (2005), arXiv: hep-th/0506029

[12] H. K. Kunduri, J. Lucietti, H. S. Reall, "Supersymmetric multi-charge AdS 5 black holes", JHEP 0604, 036 (2006), arXiv:hep-th/0601156

[13] R. Emparan, H. S. Reall, "A rotating black ring in five dimensions", Phys. Rev. Lett. 88 (2002) 101101, arXiv:hep-th/0110260

[14] H. Elvang, "A charged rotating black ring", Phys. Rev. D 68 (2003) 124016, arXiv:hep-th/0305247

[15] H. Elvang, R. Emparan, "Black rings, supertubes, and a stringy resolution of black hole non-uniqueness", JHEP 0311 (2003) 035, arXiv: hep-th/0310008

[16] R. Emparan, "Rotating circular strings, and infinite non-uniqueness of black rings", JHEP 0403, 064 (2004), arXiv: hep-th/0402149.

[17] H. Elvang, R. Emparan, D. Mateos, H. S. Reall, "Supersymmetric black rings and three-charge supertubes", Phys. Rev. D 71, 024033 (2005), arXiv:hep-th/0408120.

[18] D. Mateos, P. K. Townsend, "Supertubes", Phys. Rev. Lett. 87, 011602 (2001), arXiv: hep-th/0103030.

[19] R. Emparan, D. Mateos, P. K. Townsend, "Supergravity supertubes", JHEP 0107 (2001) 011, arXiv:hep-th/0106012

[20] D. Mateos, S. Ng, P. K. Townsend, "Tachyons, supertubes and brane/anti-brane systems", JHEP 0203, 016 (2002), arXiv:hep-th/0112054

[21] S. D. Mathur, "Where are the states of a black hole", OHSTPY-HEP-T-04-001, arXiv:hep-th/0401115.

[22] O. Lunin, S. D. Mathur, A. Saxena, "What is the gravity dual of a chiral primary?", Nucl. Phys. B 655, 185 (2003), arXiv:hep-th/0211292

[23] O. Lunin, S. D. Mathur, "AdS/CFT Duality and the Black Hole Information Paradox", Nucl. Phys. B 623 (2002) 342, arXiv: hep-th/0109154

[24] S. D. Mathur, A. Saxena, Y. K. Srivastava, "Constructing 'hair' for the three charge hole", arXiv:hep-th/0311092

[25] S. Giusto, S. D. Mathur, A. Saxena, "3-charge geometries and their CFT duals", Nucl. Phys. B 710 425-463 (2005), arXiv:hep-th/0406103

[26] S. Giusto, S. D. Mathur, A. Saxena, "Dual geometries for a set of 3-charge microstates", Nucl. Phys. B 701, 357 (2004), arXiv:hep-th/0405017.

[27] O. Lunin, "Adding momentum to D1-D5 system", JHEP 0404, 054 (2004), arXiv: hep-th/0404006

[28] I. Bena, P. Kraus, "Three charge supertubes and black hole hair", Phys. Rev. D 70, 046003 (2004), arXiv:hep-th/0402144

[29] I. Bena, "Splitting hairs of the three charge black hole", Phys. Rev. D 70 105018, (2004), arXiv:hep-th/0404073

[30] B. C. Palmer, D. Marolf, "Counting supertubes", JHEP 0406, 028 (2004), arXiv: hep-th/0403025.

[31] D. Bak, Y. Hyakutake, N. Ohta, "Phase moduli space of supertubes", Nucl. Phys. B 696, 251 (2004), arXiv: hep-th/0404104.

[32] D. Bak, Y. Hyakutake, S. Kim, N. Ohta, "A geometric look on the microstates of supertubes", Nucl. Phys. B712, 115 (2005), arXiv:hep-th/0407253

[33] S. Bellucci, E. Ivanov, S. Krivonos, "AdS/CFT Equivalence Transformation", Phys. Rev. D 66 (2002) 086001; Erratum-ibid. D 67 (2003) 049901, arXiv:hep-th/0206126.

[34] J. Shen, R. G. Cai, B. Wang, R. K. Su, "Thermodynamic Geometry and Critical Behavior of Black Holes", Int. J. Mod. Phys. A 22 (2007) 11-27, arXiv:gr-qc/0512035v1.

[35] J. E. Aman, I. Bengtsson, N. Pidokrajt, "Flat Information Geometries in Black Hole Thermodynamics", Gen. Rel. Grav. 38 (2006) 1305-1315, arXiv:gr-qc/0601119v1. 
[36] Jan E. Aman, I. Bengtsson, N. Pidokrajt, "Geometry of black hole thermodynamics", Gen. Rel. Grav. 35 (2003) 1733, arXiv:gr-qc/0304015v1.

[37] Jan E. Aman, N. Pidokrajt, "Geometry of Higher-Dimensional Black Hole Thermodynamics", Phys. Rev. D 73 (2006) 024017, arXiv:hep-th/0510139v3.

[38] T. Sarkar, G. Sengupta, B. N. Tiwari, "On the Thermodynamic Geometry of BTZ Black Holes", JHEP 0611 (2006) 015, arXiv:hep-th/0606084v1.

[39] V. Balasubramanian, Jan de Boer, V. Jejjala, J. Simon, "The Library of Babel: On the origin of gravitational thermodynamics", JHEP 0512 (2005) 006, arXiv: hep-th/0508023v2.

[40] T. Sarkar, G. Sengupta, B. N. Tiwari, "Thermodynamic Geometry and Extremal Black Holes in String Theory", JHEP 0810, 076, 2008, arXiv:0806.3513v1 [hep-th].

[41] S. Bellucci, B. N. Tiwari, "On the Microscopic Perspective of Black Branes Thermodynamic Geometry", Entropy 12, 2096, (2010), arXiv:0808.3921v1 [hep-th].

[42] B. N. Tiwari, "Sur les corrections de la géométrie thermodynamique des trous noirs", arXiv:0801.4087v1 [hep-th].

[43] T. Mishima, H. Iguchi, "New axisymmetric stationary solutions of five-dimensional vacuum Einstein equations with asymptotic flatness", Phys. Rev. D 73 (2006) 044030, arXiv:hep-th/0504018.

[44] S. Tomizawa, Y. Morisawa, Y. Yasui, "Vacuum solutions of five dimensional Einstein equations generated by inverse scattering method", Phys. Rev. D 73 (2006) 064009, arXiv:hep-th/0512252

[45] P. Figueras, "A black ring with a rotating 2-sphere", JHEP 0507, 039 (2005), arXiv: hep-th/0505244

[46] J. P. Gauntlett, J. B. Gutowski, C. M. Hull, S. Pakis, H. S. Reall, "All supersymmetric solutions of minimal supergravity in five dimensions", Class. Quant. Grav. 20, 4587 (2003), arXiv: hep-th/0209114.

[47] I. Bena, N. P. Warner, "One ring to rule them all ... and in the darkness bind them?", Adv. Theor. Math. Phys. 9 (2005) 667-701, arXiv:hep-th/0408106

[48] I. Bena, P. Kraus, "Microscopic description of black rings in AdS/CFT", JHEP 0412, 070 (2004), arXiv:hep-th/0408186

[49] P. Kraus, F. Larsen, "Attractors and black rings", Phys. Rev. D 72, 024010 (2005), arXiv:hep-th/0503219

[50] E. Witten, "String Theory Dynamics In Various Dimensions", Nucl. Phys. B 443, 85-126, (1995), arXiv:hep-th/9503124v2.

[51] M. Cyrier, M. Guica, D. Mateos, A. Strominger, "Microscopic Entropy of the Black Ring", Phys. Rev. Lett. 94, 191601 (2005), arXiv:hep-th/0411187

[52] J. M. Maldacena, A. Strominger, E. Witten, "Black Hole Entropy in M-Theory", JHEP 9712, 002 (1997), arXiv:hep-th/9711053

[53] R. Emparan, H. S. Reall, "The end of black hole uniqueness", Gen. Rel. Grav. 34, 2057 (2002).

[54] J. L. Hovdebo, R. C. Myers, "Black rings, boosted strings and Gregory-Laflamme", Phys. Rev. D 73, 084013 (2006), arXiv:hep-th/0601079.

[55] H. Elvang, R. Emparan, A. Virmani, "Dynamics and stability of black rings", arXiv:hep-th/0608076

[56] R. Gregory, R. Laflamme, "Black strings and p-branes are unstable", Phys. Rev. Lett. 70 (1993) 2837, arXiv:hep-th/9301052

[57] G. Arcioni, E. Lozano-Tellechea, "Stability and critical phenomena of black holes and black rings", Phys. Rev. D 72, 104021, (2005), arXiv:hep-th/0412118.

[58] O. J. C. Dias, "Superradiant instability of large radius doubly spinning black rings", Phys. Rev. D 73, 124035 (2006), arXiv:hep-th/0602064.

[59] G. Lopes Cardoso, B. de Wit, J. Kappeli, T. Mohaupt, "Asymptotic degeneracy of dyonic N = 4 string states and black hole entropy", JHEP 0412, 075 (2004), arXiv: hep-th/0412287

[60] G. Moore, "Introduction to modular functions and their application to 2D CFT", Spring School on Superstring Theory and Related Topics-2008,ICTP Trieste, Italy; http://cdsagenda5.ictp.trieste.it .

[61] A. Sen, "Black Holes and the Spectrum of Half-BPS States in N=4 Supersymmetric String Theory", Adv. Theor. Math. Phys. 9 (2005) 527-558, arXiv:hep-th/0504005v2. 
[62] F. Weinhold, "Metric geometry of equilibrium thermodynamics", J. Chem. Phys. 63 , 2479 (1975), DOI:10.1063/1.431689.

[63] F. Weinhold, "Metric geometry of equilibrium thermodynamics. II", Scaling, homogeneity, and generalized GibbsDuhem relations, ibid J. Chem. Phys 63 , 2484 ( 1975).

[64] G. Ruppeiner, "Riemannian geometry in thermodynamic fluctuation theory", Rev. Mod. Phys 67 (1995) 605, Erratum 68 (1996) 313.

[65] G. Ruppeiner, "Thermodynamics: A Riemannian geometric model”, Phys. Rev. A 20, 1608 (1979).

[66] G. Ruppeiner, "Thermodynamic Critical Fluctuation Theory?", Phys. Rev. Lett. 50, 287 (1983).

[67] G. Ruppeiner, "New thermodynamic fluctuation theory using path integrals", Phys. Rev. A 27,1116,1983.

[68] G. Ruppeiner and C. Davis, "Thermodynamic curvature of the multicomponent ideal gas", Phys. Rev. A 41, 2200, 1990.

[69] S. Bellucci, V. Chandra, B. N. Tiwari, "On the Thermodynamic Geometry of Hot QCD", arXiv:0812.3792v1 [hep-th].

[70] D. Tranah and P. T. Landsberg, "Collective Phenomena", 3, 81 (1980).

[71] G. Ruppeiner, "Thermodynamic curvature and phase transitions in Kerr-Newman black holes", Phy. Rev. D 78, 024016 (2008)

[72] D. Bekenstein, "Information in the holographic universe", Sci. Am. 289, No. 2, 58-65 (2003).

[73] S. D. Mathur, "The fuzzball proposal for black holes: An elementary review", Fortsch. Phys. 53 (2005) 793, arXiv: hep-th/0502050.

[74] T. Harmark, N. A. Obers, "Black holes on cylinders", JHEP 0205, 032 (2002), arXiv: hep-th/0204047.

[75] H. Elvang, R. Emparan, P. Figueras, "Non-supersymmetric black rings as thermally excited supertubes", JHEP 0502 (2005) 031, arXiv:hep-th/0412130.

[76] I. Bena, N. P. Warner, "Bubbling supertubes and foaming black holes", Phys. Rev. D74 (2006) 066001, arXiv:hep-th/0505166.

[77] I. Bena, C. W. Wang, N. P. Warner, "Black rings with varying charge density", JHEP 0603 (2006) 015, arXiv:hep-th/0411072.

[78] S. Giusto and S. D. Mathur, "Geometry of D1-D5-P bound states", Nucl. Phys. B729 (2005) 203-220, arXiv:hep-th/0409067.

[79] V. Jejjala, O. Madden, S. F. Ross and G. Titchener, "Non-supersymmetric smooth geometries and D1-D5-P bound states", arXiv:hep-th/0504181.

[80] J. C. Breckenridge, R. C. Myers, A. W. Peet, C. Vafa, "D-branes and spinning black holes", Phys. Lett. B 391, 93 (1997), arXiv:hep-th/9602065

[81] I. Bena, P. Kraus, N. P. Warner, "Black rings in Taub-NUT", Phys. Rev. D 72 (2005) 084019, arXiv:hep-th/0504142

[82] O. Lunin, S. D. Mathur, "Metric of the multiply wound rotating string", Nucl. Phys. B 610, 49 (2001), arXiv:hep-th/0105136

[83] O. Lunin, J. Maldacena, L. Maoz, "Gravity solutions for the D1-D5 system with angular momentum", arXiv:hep-th/0212210

[84] I. Bena, P. Kraus, "Microstates of the D1-D5-KK system", Phys. Rev. D 72 (2005) 025007, arXiv:hep-th/0503053.

[85] A. Dabholkar, N. Iizuka, A. Iqubal, A. Sen, M. Shigemori, "Spinning Strings as Small Black Rings", JHEP 0704:017,2007, arXiv:hep-th/0611166v2.

[86] N. Iizuka, M. Shigemori, "A note on D1-D5-J system and 5D small black ring", JHEP 0508 (2005) 100, arXiv:hep-th/0506215.

[87] A. Dabholkar, N. Iizuka, A. Iqubal, M. Shigemori, "Precision Microstate Counting Of Small Black Rings", Phys. Rev. Lett. 96, 071601 (2006), arXiv:hep-th/0511120.

[88] L. F. Alday, J. de Boer, I. Messamah, "What is the dual of a dipole?", Nucl. Phys. B 746, 29 (2006), arXiv:hep-th/0511246 
[89] H. Elvang, R. Emparan, D. Mateos, H. S. Reall, "A supersymmetric black ring", Phys. Rev. Lett. 93, 211302 (2004), arXiv:hep-th/0407065.

[90] H. Elvang, R. Emparan, D. Mateos, H. S. Reall, "Supersymmetric 4D rotating black holes from 5D black rings", JHEP 08 (2005) 042, arXiv:hep-th/0504125.

[91] J. P. Gauntlett, J. B. Gutowski, "General concentric black rings", Phys. Rev. D 71, 045002 (2005), arXiv: hep-th/0408122.

[92] I. Bena, C. W. Wang, N. P. Warner, "Plumbing the Abyss: Black Ring Microstates", JHEP 0807, 019 (2008), arXiv:0706.3786v2 [hep-th].

[93] H. Lin, O. Lunin, J.M. Maldacena, "Bubbling AdS space and 1/2 BPS geometries", JHEP 0410, 025 (2004), arXiv:hep-th/0409174.

[94] G. T. Horowitz, H. S. Reall, "How hairy can a black ring be?", Class. Quant. Grav. 22, 1289 (2005), arXiv:hep-th/0411268

[95] J. P. Gauntlett, J. B. Gutowski, "Concentric black rings", Phys. Rev. D 71, 025013 (2005), arXiv:hep-th/0408010.

[96] D. Gaiotto, A. Strominger, X. Yin, "5D black rings and 4D black holes", JHEP 02 023, (2006), arXiv:hep-th/0504126

[97] D. Marolf, A. Virmani, "A black hole instability in five dimensions?", JHEP 0511 (2005) 026, arXiv:hep-th/0505044

[98] A. Strominger, C. Vafa, "Microscopic Origin of the Bekenstein-Hawking Entropy", Phys. Lett. B 379, 99-104, (1996), arXiv:hep-th/9601029v2.

[99] H. S. Reall, "Higher dimensional black holes and supersymmetry", Phys. Rev. D 68, 024024 (2003), erratum 70, 089902 (2004), arXiv:hep-th/0211290.

[100] S. Giusto, S. D. Mathur, Y. K. Srivastava, "A microstate for the 3-charge black ring", Nucl. Phys. B 763 60-90, (2007), arXiv:hep-th/0601193

[101] P. Berglund, E. G. Gimon, T. S. Levi, "Supergravity microstates for BPS black holes and black rings", JHEP 06 (2006) 007, arXiv:hep-th/0505167.

[102] R. Emparan, D. Mateos, "Oscillator level for black holes and black rings", Class. Quant. Grav. 22, 3575 (2005), arXiv:hep-th/0506110.

[103] G. J. Galloway, R. Schoen, "A generalization of Hawking's black hole topology theorem to higher dimensions", Commun. Math. Phys. 266 571-576, (2006), arXiv:gr-qc/0509107.

[104] R. C. Myers, M. J. Perry, "Black Holes In Higher Dimensional Space-Times", Ann. Phys. 172 (1986) 304.

[105] S. W. Hawking, "Space-Time Foam", Nucl. Phys. B 144, 349 (1978). 